\title{
Renormalized Poincaré algebra for effective particles in quantum field theory
}

\author{
Stanisław D. Głazek ${ }^{\Uparrow}$ and Tomasz Masłowski \\ Institute of Theoretical Physics, Warsaw University, ul. Hoża 69, 00-681 Warsaw, Poland
}

(Dated: October 17, 2001)

\begin{abstract}
Using an expansion in powers of an infinitesimally small coupling constant $g$, all generators of the Poincaré group in local scalar quantum field theory with interaction term $g \phi^{3}$ are expressed in terms of annihilation and creation operators $a_{\lambda}$ and $a_{\lambda}^{\dagger}$ that result from a boost-invariant renormalization group procedure for effective particles. The group parameter $\lambda$ is equal to the momentum-space width of form factors that appear in vertices of the effective-particle Hamiltonians, $H_{\lambda}$. It is verified for terms order $1, g$, and $g^{2}$, that the calculated generators satisfy required commutation relations for arbitrary values of $\lambda$. One-particle eigenstates of $H_{\lambda}$ are shown to properly transform under all Poincaré transformations. The transformations are obtained by exponentiating the calculated algebra. From a phenomenological point of view, this study is a prerequisite to construction of observables such as spin and angular momentum of hadrons in quantum chromodynamics.
\end{abstract}

PACS numbers: 11.10Gh, 11.30Cp, 11.10Ef

\section{INTRODUCTION}

Complete theoretical description of relativistic particles requires understanding of their binding, which concerns how these particles form bound states and how their yet unidentified constituents might be bound, if any. The fact that potentially relevant binding mechanisms are still far from being understood is best illustrated by the current example of hadrons as built from quarks and gluons. Hadronic structure continues to pose problems with physical interpretation of phenomena such as the spin of nucleons or apparent lack of gluonic excitations in the spectrum of light hadrons. Phenomenology includes constituent quarks and gluons, partons in the infinite momentum frame, and field-theoretic concepts of QCD, but no unified understanding of the building blocks of hadrons is presently available.

A verified non-relativistic method for describing bound states in quantum mechanics is provided by the Schrödinger equation $H|\psi\rangle=E|\psi\rangle$. However, one must extend the method to include at least symmetries of special relativity. In order to combine quantum mechanics and special relativity in Hamiltonian dynamics, one needs to construct ten hermitian generators of the Poincaré algebra [1], i.e. four momenta $P^{\mu}, \mu=0,1,2,3$, and six operators $M^{\mu \nu}=-M^{\nu \mu}$ that generate rotations and boosts. The ten generators should satisfy following commutation relations,

$$
\begin{gathered}
{\left[P^{\mu}, P^{\nu}\right]=0,} \\
{\left[P^{\mu}, M^{\nu \rho}\right]=i\left(g^{\mu \nu} P^{\rho}-g^{\mu \rho} P^{\nu}\right),} \\
{\left[M^{\mu \nu}, M^{\rho \sigma}\right]} \\
=i\left(g^{\mu \rho} M^{\sigma \nu}-g^{\mu \sigma} M^{\rho \nu}+g^{\nu \rho} M^{\mu \sigma}-g^{\nu \sigma} M^{\mu \rho}\right) .
\end{gathered}
$$

\footnotetext{
*Supported by KBN grant No. 2 P03B 01618.
}

In constructing these operators one should certainly include the fact that relativistic interactions can annihilate and create particles. This phenomenon can be described using quantum field theory (QFT). But QFT leads to the problem that when one attempts to calculate the Poincaré generators using local interactions and canonical quantization, one discovers ultraviolet divergences that have to be regulated. Unfortunately, regularization of the generators destroys symmetries of the local theory and Eqs. (1.2 1.3) are not satisfied. This complication is considered by many authors an essential drawback of the canonical approach. However, if some renormalization group procedure [2] were able to remove the regularization dependent terms that violate Poincaré symmetry, the resulting quantum mechanical approach could become a candidate for the relativistic description of particle binding. The desired renormalization procedure should produce expressions for all Poincaré generators in terms of scale-dependent effective particles, instead of the bare point-like quanta. A candidate calculus for development of such effective particle scheme has been recently proposed [3] in the case of Hamiltonian operators, based on the idea of the similarity renormalization group procedure for Hamiltonian matrices [4]. The new calculus is already known to produce asymptotic freedom in the renormalized Hamiltonians for effective gluons in QCD. Present work extends the new method to all generators of the Poincaré algebra, using a simplest example of scalar particles to outline the scheme. Dynamics of particles with spin $1 / 2$ and 1 , especially in gauge theories, turn out to involve additional small- $x$ singularities that require extensive studies before conclusions can be drawn about applicability of the new method to gauge theories.

The Poincaré generators that do not depend on interactions are relatively easy to construct in QFT, since they are equal to the generators for free particles. They are called kinematical, since the transformations they generate correspond to symmetries of the space of kinematical variables that label states. The generators that in- 
volve interactions are called dynamical, for they generate transformations that involve evolution of states in time, which involves dynamics. The latter are much harder to construct in QFT because the interaction terms one obtains from local theories involve divergences. It helps to note at this point that the number of the hard-toconstruct dynamical generators depends on the form of dynamics. Three qualitatively different forms exist [1]: the well known equal-time formulation, the point form, and the front form, which is conventionally called here the light-front (LF) dynamics. These forms differ by a hyper-surface in space-time where the wave functions for a system under consideration would be defined. Different surfaces induce different expressions for the generators of the Poincaré group in QFT.

In the usual formulation, a physical system is defined on the hyper-plane of equal time, e.g. $t=0$, and the hyper-plane is invariant under six Poincaré transformations. Noether's theorem implies that there are six kinematical generators, i.e. three momenta and three generators of rotations. The remaining four generators of translations in time and three Lorentz boosts, all depend on the interactions, and they lead to the ultraviolet divergence problems. In the point form of dynamics, in which the system's evolution is traced from one space-like hyperboloid $x^{2}=$ const. to another, one has six kinematical generators, $M^{\mu \nu}$, and four dynamical, $P^{\mu}$. This form seems to have a natural relativity structure but it encounters difficulties in practice, since all momentum operators depend on interactions and momentum representation for quantum states is not easily available. In the LF dynamics, the system is defined on the hyper-plane described by equation $x \eta=0$, where $\eta$ is a zero-vector, often chosen to have components $\eta^{\mu}=\left(\eta^{0}=1, \eta^{1}=0, \eta^{2}=0, \eta^{3}=-1\right)$ so that the LF hyper-plane is given by the condition $x^{+}=x^{0}+x^{3}=0$. There are seven kinematical generators in the LF approach: $P^{+}, P^{\perp}, M^{+\perp}, M^{12}$, and $M^{+-}$, where $\perp=(1,2)$. The only dynamical generators are $P^{-}$, and $M^{-\perp}$. Moreover, $P^{+}$has exclusively positive eigenvalues, denoted by $p^{+}$, and the free single particle "energy", $p^{-}=\left(m^{2}+p^{\perp 2}\right) / p^{+}$, does not involve a square root.

These features suggest that the construction of dynamical generators could be easier in the LF dynamics than in other approaches. Quantum field theories formulated in the equal-time approach lead to Hamiltonian terms that can create particles out of the bare vacuum, as long as the created particles' three-momenta sum up to zero. These terms immediately lead to unknown ground state structures that are thought to be involved in formation of the effective degrees of freedom. The corresponding expressions for effective Poincaré generators can hardly be found before the ground state and its excitations are understood. In the regularized LF dynamics, the bare vacuum state is an eigenstate of all generators with eigenvalue zero, since creation of particles with positive momenta $p^{+}$would have to change the state momentum and translation invariance forbids that. The bare vac- uum state can be then used in a perturbative approach to build effective particle states and study their interactions. These states and interactions may turn out to contain very complicated long-wavelength components that correspond to the vacuum effects in the standard approach, but the perturbative construction can start from the physically relevant non-vacuum parts. Thus, the vacuum effects in LF dynamics are not expected to overwhelm the bound state problem from the beginning.

An important argument for considering LF dynamics comes from the fact that the seventh kinematical generator, i.e. $M^{+-}$, appears to allow boosting of states to the infinite momentum frame. Therefore, one may hope that the constituent structure of bound states in the rest frame of reference can be directly connected with the parton picture in the infinite momentum frame. A boost-invariant renormalization group procedure for effective particles in LF quantum field theory is therefore highly desired for description of high-energy collisions of particles, especially hadrons using QCD.

This work is focused on the Poincaré algebra in LF dynamics with simplest interactions that produce ultraviolet divergences, scalar fields with a cubic interaction term. Key elements of the perturbative renormalization group calculus for Poincaré algebra are related to the three-prong nature of the interactions. The same structure is encountered in all theories of physical interest. Although the scalar theory is considered unstable, the construction of generators order by order in perturbation theory never runs into the need of considering the exact ground state. Thus, the general renormalization group scheme can be studied perturbatively using scalar particles. This study exposes spin-independent features that would be a part of construction of relativistic LF dynamics of effective particles in all physically interesting theories, cf. [5].

This article is organized as follows. Section II describes regularization of the divergent bare canonical generators. Derivation of effective generators and verification of their commutation relations, are described in Section III. Section IV shows that one particle eigenstates of the Hamiltonian transform under finite Poincaré transformations as they should in a relativistic theory. An example of dynamical rotation around one of the transverse axes is presented for illustration. Section $\mathrm{V}$ concludes the paper by a brief summary of the results and some of their implications. Four Appendices contain all details needed for completeness.

\section{CANONICAL GENERATORS}

The classical local Lagrangian density

$$
\mathcal{L}=\frac{1}{2} \partial_{\mu} \phi \partial^{\mu} \phi-\frac{1}{2} m^{2} \phi^{2}-\frac{1}{3 !} g \phi^{3},
$$

provides equations of motion for the field $\phi$. Using Poincaré invariance of $\mathcal{L}$, one can introduce [6] the den- 
sity of the energy-momentum tensor,

$$
\mathcal{T}^{\mu \nu}=\frac{\partial \mathcal{L}}{\partial\left(\partial_{\mu} \phi\right)} \partial^{\nu} \phi-g^{\mu \nu} \mathcal{L},
$$

and write expressions for $P^{\mu}$ and $M^{\mu \nu}$,

$$
\begin{aligned}
P^{\mu} & =\left.\frac{1}{2} \int d^{2} x^{\perp} d x^{-} \mathcal{T}^{+\mu}\right|_{x^{+}=0}, \\
M^{\mu \nu} & =\left.\frac{1}{2} \int d^{2} x^{\perp} d x^{-}\left(x^{\mu} \mathcal{T}^{+\nu}-x^{\nu} \mathcal{T}^{+\mu}\right)\right|_{x^{+}=0}
\end{aligned}
$$

At the LF-time $x^{+}=0$, the field $\phi$ may be decomposed into Fourier components,

$$
\phi(x)=\left.\int[p]\left(e^{i p x} a_{p}^{\dagger}+e^{-i p x} a_{p}\right)\right|_{x^{+}=0},
$$

where $[p]=d p^{+} d p^{1} d p^{2} \theta\left(p^{+}\right) /\left(16 \pi^{3} p^{+}\right)$. Imposing commutation relations

$$
\left[a_{p}, a_{q}^{\dagger}\right]=p^{+} \tilde{\delta}(p-q),
$$

where $\tilde{\delta}(k)=16 \pi^{3} \delta\left(k^{+}\right) \delta\left(k^{1}\right) \delta\left(k^{2}\right)$, inserting Eq. 2.5) into Eqs. (2.3) and (2.4), putting all creation operators to the left of all annihilation operators, and dropping all singular terms that result from the ordering, one obtains a set of heuristic expressions for Poincaré generators that are listed in Appendix A. If the interactions are absent, i.e. $g=0$, these generators satisfy all commutation relations (1.1) - (1.3). However, when the interaction is present, i.e. for $g \neq 0$, products of $P^{-}, M^{-1}$ and $M^{-2}$ produce divergent operators. The divergences result from summation over intermediate states of unlimited free energies. To provide meaning to the otherwise divergent products, one has to impose some cutoff on the kinematical momentum variables so that the energy range in the summation becomes finite. Introduction of such a cutoff is called regularization. In the LF dynamics, the regularization of $P^{-}, M^{-1}$ and $M^{-2}$ is introduced through artificially inserted factors $r_{\Delta}$ that multiply interaction vertices and fall off to zero when particle momenta change in a single interaction by more than certain cutoff parameter $\Delta$.

Let $\hat{O}_{n}$ denote a coefficient of $g^{n}$ in the operator $\hat{O}$, so that $\hat{O}=\hat{O}_{0}+g \hat{O}_{1}+g^{2} \hat{O}_{2}+\ldots$. In this convention, the regulated bare generators contain only terms denoted by $P_{\Delta 1}^{-}$and $M_{\Delta 1}^{-j}$. They are written as follows (see Appendix $\mathrm{A}$ for details of the notation).

$$
\begin{aligned}
P_{\Delta 1}^{-} & =\frac{1}{2} \int[123] \tilde{\delta} r_{\Delta} a_{1}^{\dagger} a_{2}^{\dagger} a_{3}+\text { h.c. } \\
M_{\Delta 1}^{-j} & =\frac{i}{2} \int[123]\left(\frac{\partial}{\partial p_{3}^{j}} \tilde{\delta}\right) r_{\Delta} a_{1}^{\dagger} a_{2}^{\dagger} a_{3}+\text { h.c. } .
\end{aligned}
$$

The ultraviolet regularization factors are chosen here in the form $r_{\Delta}=\exp \left(-\kappa_{12}^{\perp 2} / \Delta^{2}\right)$, where $\kappa_{12}^{\perp}=\left(p_{1}^{\perp} p_{2}^{+}-\right.$ $\left.p_{2}^{\perp} p_{1}^{+}\right) /\left(p_{1}^{+}+p_{2}^{+}\right)$is a relative transverse momentum of particles 1 and 2 . When regularization is being removed, $\Delta \rightarrow \infty$ and the regulating factors $r_{\Delta}$ tend to 1 for all finite $\kappa_{12}$.

The particular choice made here for $r_{\Delta}$ is not unique and if the regularization is to be removed, it should not matter how it is introduced. Nevertheless, in the intermediate steps of deriving the regularization-independent renormalized theory, one prefers to use regularizations that make the procedure of removing the cutoff dependence least complicated. The choice made here for $r_{\Delta}$ is dictated by experience with various other factors and convenience of using them in calculations. The chosen factor preserves the kinematical symmetries of LF dynamics and maintains factorization of longitudinal and transverse momenta. The exponential function is usefully compatible with analytic integration of the renormalization group equations. An example of considerations that matter in choosing $r_{\Delta}$ is provided by a natural candidate factor in LF dynamics: $\exp \left(-\mathcal{M}_{12}^{2} / \Delta^{2}\right)$, where $\mathcal{M}_{12}^{2}=\left(p_{1}+p_{2}\right)^{2}$ is a free invariant mass of particles 1 and 2 , squared. That choice introduces $\Delta$-dependence even in tree-like interaction terms that involve small values of $p_{1}^{+}$or $p_{2}^{+}$for finite external momenta. This feature is not helpful in the scalar theory and the present article is simplified by avoiding it, independently of whether regularization factors that depend on invariant masses can or cannot help in constructing effective dynamics in gauge theories where small- $p^{+}$singularities in tree-like terms are common.

Although the regularization renders finite candidates for the interaction-dependent generators, the cutoff destroys formal Poincaré symmetry of local theory. In the model case here, three Poincaré algebra commutation relations are violated by cutoff-dependent terms of order $g$ and $g^{2}$. Namely, the commutators

$$
B_{\Delta}^{j}=\left[P_{\Delta}^{-}, M_{\Delta}^{-j}\right],
$$

for $j=1,2$ and

$$
B_{\Delta}^{12}=\left[M_{\Delta}^{-1}, M_{\Delta}^{-2}\right],
$$

should be equal to zero in the correct algebra, while the regulating factors cause that these commutators are not equal to zero. Their structure is described in Appendix A.

The problem of Poincaré symmetry violation is solved in the present work in perturbation theory up to the terms order $g^{2}$ using a renormalization group procedure described in next sections. The procedure is used to calculate counterterms and derive finite effective generators in the limit $\Delta \rightarrow \infty$. The virtue of the procedure, however, is not merely that the resulting algebra can be satisfied in perturbation theory, but also that the resulting effective particle dynamics involves only finite momentum changes in all interactions. The scale of allowed momentum changes is determined by the parameter that labels operators derived by solving the renormalization group 
equations. Thus, the procedure produces Poincaré generators expressed in terms of effective particles that are quite different from the bare ones and cannot emit or absorb momenta comparable with $\Delta$. Note that one could also attempt to remove the symmetry violating terms of order $g^{2}$ by adding ad hoc terms $\tilde{B}_{\Delta}^{j}$ to $M_{\Delta}^{-j}$, with $\left[P_{0 \Delta}^{-}, \tilde{B}_{\Delta}^{j}\right]=-B_{\Delta}^{j}$, and try to keep working with bare particles and momentum transfers in interactions among them ranging up to $\Delta \rightarrow \infty$. This is not what the renormalization group procedure is about or leads to. For example, the counterterms one obtains in second order perturbation theory correct particle masses, instead of adding terms like $\tilde{B}_{\Delta}^{j}$.

\section{EFFECTIVE GENERATORS}

The effective Poincaré group generators are found using the renormalization group procedure [3] that provides means for finding counterterms in the initial bare Hamiltonian and defines annihilation and creation operators for effective particles, $a_{\lambda}$ and $a_{\lambda}^{\dagger} . \quad \lambda$ is the renormalization group parameter. In the initial regularized theory $\lambda=\infty$. Detailed formulas of the effective particle calculus used in this work are given in Appendices B and $\mathrm{Q}$. This Section provides a qualitative introduction to the method and extends it to all generators of the Poincaré algebra. Operators $a_{\lambda}$ and $a_{\lambda}^{\dagger}$ are generally denoted by $q_{\lambda}$ wherever it does not matter which of the operators is spoken about. The bare particle creation and annihilation operators, $q_{\infty}$, are shortly denoted by $q$.

The operators $q_{\lambda}$ are used to define effective particle basis states in the Fock space and to write the Poincaré algebra in the form easy to use in that basis, i.e. in terms of superpositions of products of $q_{\lambda}$. The generators written in terms of $q_{\lambda}$ are also called effective. They turn out to be free from the Poincaré symmetry violating cutoff effects in a perturbative theory in the limit $\Delta \rightarrow \infty$. The reason, to be explained below, is that they do not directly couple effective particle states with small and large invariant masses. This feature is analogous but not identical to removal of large energy jumps in the original similarity renormalization group procedure [4]. The main difference is that the effective generators are able to produce larger jumps than the effective time-evolution generator itself. This feature will be explained later, too.

The required expression for $q=q\left(q_{\lambda}\right)$ is found from equations that determine renormalized Hamiltonians $P_{\lambda}^{-}$. The initial regularized Hamiltonian $P_{\Delta}^{-}$that includes unknown counterterms, is originally expressed in terms of bare operators $q$. One assumes that $q$ and $q_{\lambda}$ are connected by a unitary transformation that preserves quantum numbers labeling $q$ and $q_{\lambda}$,

$$
q=\mathcal{U}_{\lambda}^{\dagger} q_{\lambda} \mathcal{U}_{\lambda}
$$

and one solves the renormalization group equations from Appendix B to derive effective Hamiltonians, i.e. $P_{\lambda}^{-}$expressed in terms of effective operators $q_{\lambda}$ for all values of $\lambda$. This procedure provides $\mathcal{U}_{\lambda}$ and Appendix $\mathrm{C}$ explains how.

The effective Hamiltonians $P_{\lambda}^{-}$contain vertex form factors $f_{\lambda}$ in interaction terms ( $\lambda$ is the width of the form factors in momentum space). Therefore, the Hamiltonians do not directly couple states of effective particles with invariant masses that differ by more than about $\lambda$ and the interaction terms need to act at least about $\Delta / \lambda$ times to reach masses order $\Delta$ starting from finite masses. Thus, if the effective Hamiltonian terms for finite momenta have a limit when $\Delta \rightarrow \infty$, the resulting theory is free from the regularization dependence to all orders of perturbation theory. Therefore, the counterterms required in $P_{\Delta}^{-}$can be found from the condition that for finite relative momenta coefficients of products of $q_{\lambda}$ in the operators $P_{\lambda}^{-}$are independent of $\Delta$ when $\Delta \rightarrow \infty$. Similar condition is used for finding counterterms in all generators.

Once the function $q\left(q_{\lambda}\right)$ is found from equations for $P_{\lambda}^{-}$, see Appendix $\mathrm{Q}$, all other Poincaré generators are expressed in terms of $q_{\lambda}$ by a plain substitution. All divergences in the resulting expressions are identified and counterterms in the bare generators are introduced as required by the cancellation of the divergences in the generators expressed in terms of $q_{\lambda}$. In the scalar theory, it turns out that only mass counterterms are required in terms of order $g^{2}$. Once the counterterms in the initial generators are found, one takes the limit $\Delta \rightarrow \infty$ in the expressions written in terms of $q_{\lambda}$. The resulting operators constitute the effective Poincaré algebra. The key point of the whole construction is that the finite scale $\lambda$ in vertex form factors destroys the ability of $n$-th order Poincaré generators to change momenta of effective particles by more than $n \lambda$ and the limit $\Delta \rightarrow \infty$ is well defined in perturbation theory in the effective Fock space basis.

Since the effective interactions are smoothed by the form factors of width $\lambda$, the time evolution in physical processes characterized by some momentum transfer scale $Q$ is most naturally described by the effective Hamiltonian $H_{Q}\left(q_{Q}\right)$. Namely, using $H_{\lambda}\left(q_{\lambda}\right)$ with $\lambda \gg Q$, one would introduce many tiny details whose net effect could presumably be absorbed into renormalization of effective interaction parameters, as in the case of logarithms of the ratio $\lambda / Q$ that contribute to the running coupling constant. On the other hand, using $\lambda \ll Q$, one would have to unnecessarily consider multiple effective interactions to build momentum transfers on the order of $Q$ through about $Q / \lambda$ small steps, each transferring at most about $\lambda$. To work with the effective particles with $\lambda=Q$ and to have control on transformation properties of physical states, it is desirable to have all generators written in terms of $q_{Q}$.

Mathematically, any bare generator with added counterterms, $\tilde{A}_{\infty}(q)=A_{\infty}+X_{\infty}$, is expressed in terms of effective creation and annihilation operators using formula 


$$
\tilde{A}_{\infty}\left[q\left(q_{\lambda}\right)\right]=\tilde{A}_{\lambda}\left(q_{\lambda}\right) .
$$

The symbol is used to indicate that the regularization cutoff parameter $\Delta$ is still kept finite at this stage of the calculation. However, once the counterterms $X_{\infty}$ are found and the effective generators calculated, one can take the limit $\Delta \rightarrow \infty$ by putting $r_{\Delta}=1$ in the effective generators. Thus, the effective generators are given by the formula

$$
A_{\lambda}\left(q_{\lambda}\right)=\left.\tilde{A}_{\lambda}\left(q_{\lambda}\right)\right|_{r_{\Delta}=1} .
$$

Results obtained from this formula are described below.

Although operators $q$ expressed in terms of $q_{\lambda}$ contain terms of order $1, g, g^{2}$ and higher, all seven effective kinematical generators appear unchanged in form, as if they continued to be independent of interactions. Namely, the only change that occurs in them is that the bare creation and annihilation operators are transformed into the operators for effective particles of scale $\lambda$. The coefficients are not changed and they remain equal to the coefficients found in the interacting canonical theory as well as in the free theory. This result follows from kinematical symmetries of LF dynamics, which are preserved in the renormalization group equations.
On the other hand, the interaction terms in the dynamical generators are changed considerably and contain products of form factors $f_{\lambda}$ that limit momentum transfers by $\lambda$ or $2 \lambda$. The factor of two results from a convolution of two first order terms, each containing $f_{\lambda}$. The appearance of form factors in this pattern is verified here only in terms of order $g$ and $g^{2}$. Nevertheless, on the basis of these two cases and observed regularities that appear along with derivatives over particle momenta in the generators of rotations, one is compelled to consider it plausible, though not proved yet, that in higher orders, say $n$-th, the form factors will appear following the same pattern and limit momentum transfers by $n \lambda$. This explains why the generators of rotations can produce jumps larger than the jumps allowed by $P_{\lambda}^{-}$itself.

In the interaction terms order $g$, the only change besides introduction of form factors $f_{\lambda}$ is the replacement of $q$ by $q_{\lambda}$. In terms order $g^{2}$, new features appear. Namely, there emerge several additional interactions with products of more than three effective operators, in distinction from Eqs. (2.3) and (2.4) in the bare canonical theory where at most three bare operators are present. In addition, terms order $g^{2}$ involve mass renormalization. The complete result, derived using Appendices B and $\mathrm{Q}$, is following, see also Fig 1 . and 2 .

$$
\begin{aligned}
P_{\lambda 1}^{-}= & f_{\lambda} P_{1}^{-}\left(q \rightarrow q_{\lambda}\right), \\
P_{\lambda 2}^{-}= & \int[p] \frac{\delta m_{\lambda}^{2}}{p^{+}} a_{\lambda p}^{\dagger} a_{\lambda p} \\
& +\int[1234]\left[f_{\lambda} \tilde{\delta} V_{\lambda}^{22} a_{\lambda 1}^{\dagger} a_{\lambda 2}^{\dagger} a_{\lambda 3} a_{\lambda 4}+\left(f_{\lambda} \tilde{\delta} V_{\lambda}^{31} a_{\lambda 1}^{\dagger} a_{\lambda 2}^{\dagger} a_{\lambda 3}^{\dagger} a_{\lambda 4}+\text { h.c. }\right)\right],
\end{aligned}
$$

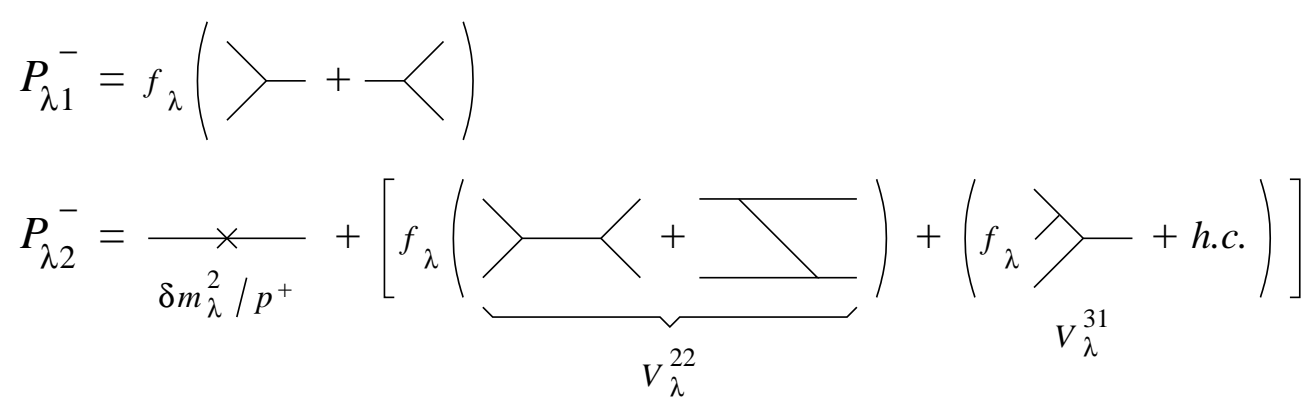

FIG. 1: Structure of the first and second order interaction terms in $P_{\lambda}^{-}$, Eqs. 3.4 ) and 3.5 . 


$$
\begin{aligned}
& M_{\lambda 1}^{-j}=f_{\lambda} M_{1}^{-j}\left(q \rightarrow q_{\lambda}\right), \\
& M_{\lambda 2}^{-j}=i \int[p] \frac{\delta m_{\lambda}^{2}}{p^{+}}\left(\frac{\partial a_{\lambda p}^{\dagger}}{\partial p^{j}}\right) a_{\lambda p} \\
& +i \int[1234] \frac{1}{p_{34}^{-}-p_{12}^{-}}\left[f_{\lambda} D^{j}\left(\tilde{\delta} V_{\lambda}^{22}\right)+\tilde{\delta} \tilde{V}_{\lambda}^{j 22}\right] a_{\lambda 1}^{\dagger} a_{\lambda 2}^{\dagger} a_{\lambda 3} a_{\lambda 4} \\
& +\left\{i \int[1234] \frac{1}{p_{4}^{-}-p_{123}^{-}}\left[f_{\lambda} D^{j}\left(\tilde{\delta} V_{\lambda}^{31}\right)+\tilde{\delta} \tilde{V}_{\lambda}^{j 31}\right] a_{\lambda 1}^{\dagger} a_{\lambda 2}^{\dagger} a_{\lambda 3}^{\dagger} a_{\lambda 4}+\text { h.c. }\right\} . \\
& \left.M_{\lambda 1}^{-j}=f_{\lambda}(\rangle^{j}+\stackrel{j}{j}\right\rangle \\
& M_{\lambda 2}^{-j}=\frac{x^{j}}{\delta m_{\lambda}^{2} / p^{+}}+\frac{1}{\operatorname{Den} .}\left[f_{\lambda} D^{j}(\underbrace{亠_{\sigma_{\lambda}^{j 22}}^{+\backslash}}_{V_{\lambda}^{22}}]\right. \\
& \left.+\{\frac{1}{\text { Den. }}\left[f_{\lambda} D^{j}(\rangle_{V_{\lambda}^{31}}\right)+\overbrace{\tilde{V}_{\lambda}^{j 31}}]+\text { h.c. }\right\}
\end{aligned}
$$

FIG. 2: Structure of the first and second order interaction terms in the generators $M_{\lambda}^{-j}, j=1,2$; Eqs. (3.6) and (3.7). The superscript $j$ of $M_{\lambda}^{-j}$ is carried by the differential operators on the right-hand side of the figures (contained also in the functions $\tilde{V}_{\lambda}^{j 22}$ and $\left.\tilde{V}_{\lambda}^{j 31}\right)$.

The above expressions involve symbols $D^{j}=$ $\sum_{l=1}^{4}\left(p_{l}^{-} \partial / \partial p_{l}^{j}+2 p_{l}^{j} \partial / \partial p_{l}^{+}\right)$and $p_{i j \ldots n}^{-}=$ $p_{i}^{-}+p_{j}^{-}+\cdots+p_{n}^{-}$. Symbols $V_{\lambda}^{22}, \tilde{V}_{\lambda}^{j 22}, V_{\lambda}^{31}$, and $\tilde{V}^{j 31}$, are explained in Appendices B and Q , Eqs. (B20), $(\overline{B 22}),(\mathrm{B} 21)$, and (B23), respectively.

The terms $\tilde{V}_{\lambda}^{j 22}$ and $V_{\lambda}^{j 31}$ in $M_{\lambda 2}^{-j}$ have no counterparts in $P_{\lambda 2}^{-}$. These terms turn out to cancel similar terms that appear in the Poincaré algebra commutators, e.g. they cancel $\lambda$-dependent terms in $\left[P_{\lambda 1}^{-}, M_{\lambda 1}^{-j}\right]$ in the commutator $\left[P_{\lambda}^{-}, M_{\lambda}^{-j}\right]$ evaluated up to order $g^{2}$. The standard steps of canonical quantization of local field theory, regularization, and incorporation of counterterms for $\Delta \rightarrow \infty$ alone, would not be able to produce the new terms with finite $\lambda$. The latter are derived here through application of the renormalization group procedure for effective particles, which is an additional step in constructing a finite theory.

The renormalized effective mass correction, $\delta m_{\lambda}^{2}$ in $P_{\lambda 2}^{-}$ and $M_{\lambda 2}^{-j}$, is given by the formula

$$
\delta m_{\lambda}^{2}=\delta m_{0}^{2}+\frac{1}{32 \pi^{2}} \int_{0}^{1} d x \int_{\mathcal{M}_{0}^{2}}^{\infty} d \mathcal{M}^{2} \frac{f_{\lambda}^{2}-f_{\lambda_{0}}^{2}}{\mathcal{M}^{2}-m^{2}},
$$

where $\mathcal{M}_{0}^{2}=m^{2} /[x(1-x)]$. The free parts of the mass counterterms in $P_{\lambda 2}^{-}$and $M_{\lambda 2}^{-j}$ have to be chosen equal to one value $\delta m_{0}^{2}$, for a chosen $\lambda_{0}$, to satisfy the Poincaré algebra commutation relations.

The effective generators defined above satisfy all commutation relations (1.1)-(1.3) in terms of order $1, g$, and $g^{2}$ for arbitrary values of the form factor width $\lambda$. This result is checked by explicit calculation. The key features that allow the algebra to close for arbitrary finite $\lambda$, even as small as the mass scale in the theory, are that (1) the form factor $f_{\lambda}$ is introduced by integration of renormalization group equations for a unitary rotation of operators $q$, which preserves commutation relations, (2) the argument of $f_{\lambda}$ is given by squares of sums of free particle four-momenta that are invariant under all ten Poincaré transformations with $g=0$, and (3) the initial condition from local field theory provides an algebra that is violated only by the regularization, which is lifted when one takes the limit $\Delta \rightarrow \infty$ after calculating the counterterms. It is also interesting that the Lorentz symmetry generators come out correctly thanks to the preservation of the translational symmetry throughout the scheme. 


\section{ROTATION OF PHYSICAL STATES}

It is well known from earlier works on the effective particle calculus, that the second-order two-particle scattering matrix that results from the LF old-fashioned Hamiltonian approach using $P_{\lambda}^{-}$, is fully covariant. The only contributions to the second-order come from tree diagrams with two effective vertices and from an effective two-body interaction. These contributions combine to the same result that is obtained from the Feynman rules. At the same time no dependence on $\lambda$ appears in the final formula of order $g^{2}$ for scattering amplitudes, precisely as it is required by the renormalization group procedure. But at such low order of perturbation theory no mass and no coupling constant renormalization are involved in the scattering amplitudes. The amplitudes that involve perturbative renormalization group effects are of order $g^{4}$, and higher. Therefore, the results obtained in previous sections for terms order $1, g$, and $g^{2}$, must be extended up to the fourth order terms to study symmetries of the scattering matrix calculated in the effective theory and to verify applicability of the constructed interacting algebra to gedanken physical processes with scalar particles.

However, even in the second order analysis there exists a test that the effective algebra has to pass independently of the higher order terms. Namely, scalar eigenstates of the effective Hamiltonian $H_{\lambda}\left(q_{\lambda}\right)$ that are used to construct single-particle incoming and outgoing states, should transform accordingly to the rules of representation theory for the Poincaré group, which state that [7]

$$
U(\Lambda)|\Psi(p)\rangle=\left|\Psi\left(p_{\Lambda}\right)\right\rangle .
$$

This equation means that a unitary operator $U$ representing a Poincaré transformation $\Lambda$ changes a physical scalar single-particle state of the arbitrary fourmomentum $p$ into a physical single-particle state of the four-momentum $p_{\Lambda}=\Lambda p$. This is a testing requirement because $p^{-}$component of the momentum is an eigenvalue of the full interacting Hamiltonian $H_{\lambda}\left(q_{\lambda}\right)$, and $U(\Lambda)$ involves interactions through the interaction-dependent Poincaré generators. Note that one has to know explicit expressions for $U(\Lambda)$ for all Poincaré transformations in order to be able to fully verify symmetry of observables calculated in effective theories with some value of $\lambda$. In particular, all physical single-particle states should actually be labeled only by the values of kinematical variables $p^{+}$and $p^{\perp}$, while the eigenvalue $p^{-}$should depend on $p^{+}$ and $p^{\perp}$ as in Eq. (4.2) below. By construction, this condition holds for states obtainable from a selected physical single-particle state using the seven Poincaré transformations that are kinematical in LF dynamics, and by translations in "time" $x^{+}$. It should also hold for states that result from rotations. Since rotations are dynamical, they could, in principle, generate anomalies due to the fact that the LF Hamiltonian approach to quantum field theory does distinguish a frame of reference in which the quantum states and operators are defined, and explicit Poincaré symmetry is not kept. In these circumstances, it is interesting to see how the rotational symmetry of the spectrum is realized in a fixed effective particle Fock space basis.

In this Section, the physical one-particle states are constructed by solving equation $H_{\lambda}\left(q_{\lambda}\right)|\psi\rangle=E|\psi\rangle$ in perturbation theory up to terms of order $g^{2}$. Then, the generators $A_{\lambda}\left(q_{\lambda}\right)$ are exponentiated to obtain finite Poincaré group elements and the latter are applied to the oneparticle eigenstates to see if they transform properly, keeping track of all terms order $1, g$, and $g^{2}$. The general procedure is presented on the example of rotations about one transverse axis. The construction shows in detail how the interacting angular momentum and rotation operators by finite angles act in the LF Fock space. Operators representing other Poincaré transformations are derivable in the same way and verification of their properties does not need to be re-produced here explicitly.

Eigenstates of $P_{\lambda}^{-}$with eigenvalues $p_{p h y s .}^{-}=\left(p^{\perp 2}+\right.$ $\left.m_{\text {phys. }}^{2}\right) / p^{+}$are determined by the eigenvalue equation,

$$
P_{\lambda}^{-}|\Psi(p)\rangle=p_{\text {phys. }}^{-}|\Psi(p)\rangle
$$

Their effective particle Fock space basis expansion has the form,

$$
\begin{aligned}
& |\Psi(p)\rangle=N\left[\left|\Psi_{0}(p)\right\rangle\right. \\
& \left.+g\left|\Psi_{1}(p)\right\rangle+g^{2}\left|\Psi_{2}^{11}(p)\right\rangle+g^{2}\left|\Psi_{2}^{2}(p)\right\rangle+\cdots\right],
\end{aligned}
$$

where $N$ is a normalization factor, which follows from the condition

$$
\left\langle\Psi(p) \mid \Psi\left(p^{\prime}\right)\right\rangle=p^{+} \tilde{\delta}\left(p-p^{\prime}\right) .
$$

$N$ does not change under Poincaré transformations and it factors out from their analysis. However, $N$ depends on interactions for given $\lambda$, because it compensates for the size of components generated by $P_{\lambda}^{-}$. Therefore, if one were interested in expanding a normalized state in a series of powers of $g, N$ would contribute.

In Eq. (4.3), the state component with subscript 0 denotes terms with wave-functions order $g^{0}$, subscript 1 indicates terms with wave-functions of order $g^{1}$ with the factor $g$ written explicitly in front, subscript 2 indicates terms with wave-functions of order $g^{2}$ with the factor $g^{2}$ written explicitly in front, and so on. It is necessary to distinguish wave-functions and states in the perturbative expansion since the effective particle basis states depend on interactions and also have an expansion in powers of $g$ when expressed in terms of the bare particle basis states. Since the latter expansion is already included in the effective creation operators $a_{\lambda}^{\dagger}$, the expansion for the wave-functions in the effective basis is separated. This separation is also related to the fact that in higher order analysis, including renormalization group evolution of the coupling constant $g_{\lambda}$ that starts with terms of order $g^{3}$, the wave-functions would be expanded in a series of powers of $g_{\lambda}$, or found numerically in a non-perturbative calculation using $H_{\lambda}\left(q_{\lambda}\right)$. In the present work that includes only terms up to order $g^{2}$, 
no difference between $g$ and $g_{\lambda}$ is visible. However, the expansion of wave-functions should be thought about as expansion in a series of powers of $g_{\lambda}$, not the initial $g$.

The superscripts in Eq. (4.3) indicate the origin of terms in perturbation theory using $H_{\lambda}\left(q_{\lambda}\right)$. Terms with superscript 11 result from double action of Hamiltonian terms order $g$ on the component $\left|\Psi_{0}\right\rangle$, i.e. they originate from the second order perturbation theory for wavefunctions in the effective dynamics of scale $\lambda$. In contrast, terms with superscript 2 result from single action of second order terms in the effective Hamiltonian of scale $\lambda$. In other words, the latter terms should be thought about as proportional to $g_{\lambda}^{2}$ and coming from a single action of a second-order interaction term from $H_{\lambda}\left(q_{\lambda}\right)$ on the component $\left|\Psi_{0}\right\rangle$, i.e. they originate from the first order perturbation theory for wave-functions in the effective dynamics of scale $\lambda$. As further example of the superscript notation, the component $\left|\Psi_{1}\right\rangle$ could carry a superscript 1 , which is omitted.

Direct calculation gives the following results for physical one-particle states, starting from one-effectiveparticle state,

$$
\left|\Psi_{0}(p)\right\rangle=a_{\lambda p}^{\dagger}|0\rangle
$$

First order perturbation theory gives terms $\left|\Psi_{1}\right\rangle$ that contribute in order $g$,

$$
\left|\Psi_{1}(p)\right\rangle=\frac{1}{2} \int[12] \tilde{\delta}\left(p-p_{12}\right) \frac{f_{\lambda}}{p^{-}-p_{12}^{-}}|12\rangle
$$

where $|12\rangle=a_{\lambda 1}^{\dagger} a_{\lambda 2}^{\dagger}|0\rangle$, and terms $\left|\Psi_{2}^{2}\right\rangle$ that contribute in order $g^{2}$,

$$
\left|\Psi_{2}^{2}(p)\right\rangle=\int[123] \tilde{\delta}\left(p-p_{123}\right) \frac{f_{\lambda}}{p^{-}-p_{123}^{-}} V_{\lambda}^{31}|123\rangle
$$

where $|123\rangle=a_{\lambda 1}^{\dagger} a_{\lambda 2}^{\dagger} a_{\lambda 3}^{\dagger}|0\rangle$. Second order perturbation theory gives $\left|\Psi_{2}^{11}\right\rangle$ that contributes in order $g^{2}$,

$$
\left|\Psi_{2}^{11}(p)\right\rangle=\int[123] \frac{\tilde{\delta}\left(p-p_{123}\right) f_{12} f_{(12) 3}}{2 p_{12}^{+}\left(p^{-}-p_{123}^{-}\right)\left(p^{-}-p_{(12) 3}^{-}\right)}|123\rangle .
$$

Here, $p_{(12) 3}^{-}$stands for $\left(p_{12}^{\perp}+m^{2}\right) / p_{12}^{+}+p_{3}^{-}$, which means that the total three-momentum of particles 1 and $2, p_{12}^{\perp}$ and $p_{12}^{+}$, is turned into a one-particle momentum and the corresponding $p^{-}$is evaluated using mass $m$. The same convention for $p_{(12) 3}^{-}$is used in evaluating $f_{(12) 3}$. The subscripts $i j$ in $f_{i j}$, with (12) understood as one particle subscript such as $i$, indicate that one of the invariant masses in the definition $(\mathrm{B} 6)$ is given by $\left(p_{i}+p_{j}\right)^{2}$, while the other by $m^{2}$. The eigenvalue Eq. (4.2) leads to, cf. Eq. (D29),

$$
m_{\text {phys. }}^{2}=m^{2}+g^{2} \delta m_{0}^{2}+\frac{g^{2}}{32 \pi^{2}} \int_{0}^{1} d x \int_{\mathcal{M}_{0}^{2}}^{\infty} d \mathcal{M}^{2} \frac{f_{\lambda_{0}}^{2}}{\mathcal{M}^{2}-m^{2}}=m^{2}+g^{2} \delta \tilde{m}_{0}^{2}
$$

This result shows that $m_{\text {phys. }}^{2}$ is independent of $\lambda$, as required for a physical quantity.

The next step is to show that effective Poincaré transformations obtained by exponentiation of the algebra derived in Section III, properly transform single physical particle states up to terms of order $g^{2}$ in the perturbative series. This is done below on the example of rotations around transverse axis number 1. As already mentioned, other rotations and all other Poincaré transformations are verified term by term in the series expansion in powers of $g$ using the same scheme.
The effective generator of rotations around the transverse axis number 1 is given by

$$
J_{\lambda}^{1}=\frac{1}{2}\left(M_{\lambda}^{-2}-M_{\lambda}^{+2}\right)
$$

where $M_{\lambda}^{-2}$ depends on interactions, see Eqs. (3.6) and (3.7). Physically, finite rotations around axis number 1 by an arbitrary angle $\alpha$ change the standard spatial three-momenta, $\vec{p}_{\text {phys. }}=\left(p^{1}, p^{2}, p^{3}\right)$, so that

$$
\left(p^{1}, p^{2}, p^{3}\right) \longrightarrow\left(p^{1}, p^{2} \cos \alpha-p^{3} \sin \alpha, p^{3} \cos \alpha+p^{2} \sin \alpha\right)=: \vec{p}_{p h y s . \alpha}
$$

and $p_{\text {phys. }}^{0}$ remains unchanged, so that the condition that

$p_{\text {phys. }}^{2}=m_{\text {phys. }}^{2}$ is preserved: $p_{\text {phys. } \alpha}^{2}=m_{\text {phys. }}^{2}$. Note 
that $p^{3}=\left(p^{+}-p_{\text {phys. }}^{-}\right) / 2$. The quantum operator that represents this finite rotation is given by

$$
U(\alpha)=e^{-i \alpha J_{\lambda}^{1}} .
$$

One verifies the rule (4.1) by checking if the state

$$
\left|\Psi_{\alpha}(p)\right\rangle:=U(\alpha)|\Psi(p)\rangle
$$

whose expansion in perturbation theory follows from the expression

$$
\begin{aligned}
& \left|\Psi_{\alpha}(p)\right\rangle=N\left[U_{0}(\alpha)+g U_{1}(\alpha)+g^{2} U_{2}(\alpha)+o\left(g^{3}\right)\right] \\
& \quad \times\left[\left|\Psi_{0}(p)\right\rangle+g\left|\Psi_{1}(p)\right\rangle+g^{2}\left|\Psi_{2}(p)\right\rangle+o\left(g^{3}\right)\right],
\end{aligned}
$$

satisfies the relation

$$
\left|\Psi_{\alpha}(p)\right\rangle=\left|\Psi\left(p_{\text {phys. } \alpha}\right)\right\rangle .
$$

Details of the calculation are given in Appendix D. The results can be written in a concise form by introducing a new symbol $p_{\alpha}$ to denote the rotated momentum that has the same components $p^{+}$and $p^{\perp}$ as $p_{\text {phys. }}$. before the rotation, while the rotated components $p_{\alpha}^{+}$and $p_{\alpha}^{\perp}$ are calculated by applying the rotation by angle $\alpha$ to the four-momentum with the minus component given by condition $p^{-}=\left(p^{\perp 2}+m^{2}\right) / p^{+}$, i.e. the condition $p^{2}=$ $m^{2}$ instead of $p^{2}=m_{\text {phys. }}^{2}$. Thus, $p_{\alpha}$ is obtained from the same relation (4.11) that defines $p_{\text {phys. } \alpha}$, but with $m_{\text {phys. }}^{2}$ replaced by $m^{2}$, see Eq. (D11). One obtains,

$$
\begin{aligned}
& \left|\Psi_{\alpha 0}(p)\right\rangle=\left|\Psi_{0}\left(p_{\alpha}\right)\right\rangle, \\
& \left|\Psi_{\alpha 1}(p)\right\rangle=\left|\Psi_{1}\left(p_{\alpha}\right)\right\rangle,
\end{aligned}
$$

$$
\begin{aligned}
& \left|\Psi_{\alpha 2}(p)\right\rangle=\left|\Psi_{2}\left(p_{\alpha}\right)\right\rangle \\
& +\frac{\delta m_{\lambda}^{2}-\delta \tilde{m}_{\lambda}^{2}}{p_{\alpha}^{+}}\left(\frac{\sin \alpha}{2} \frac{\partial}{\partial p^{2}}-\sin ^{2} \frac{\alpha}{2} \frac{\partial}{\partial p^{+}}\right) a_{\lambda p_{\alpha}}^{\dagger}|0\rangle,
\end{aligned}
$$

where $\delta \tilde{m}_{\lambda}^{2}$ is given by Eq. (D29).

The change of $p$ to $p_{\alpha}$ in the rotated components in Eqs. (4.16) and (4.17), agrees with the rule (4.1). Eq. (4.18) also agrees with that rule but the second term in $\left|\Psi_{\alpha 2}(p)\right\rangle$ on the right-hand side of Eq. (4.18) requires explanation. It comes from terms that are shown graphically in Fig. 3. To check that Eq. (4.18) is correct,

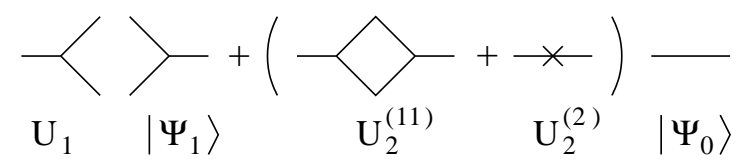

FIG. 3: Graphical representation of terms that contribute to the dynamical mass effects in rotations, see Eq. (4.18) and Appendix D3.

one needs to replace $p$ in $\left|\Psi_{0}(p)\right\rangle$ with $p_{\text {phys. } \alpha \text {. When }}$ the correction order $g^{2}$ in the physical mass, i.e. $g^{2} \delta \tilde{m}_{0}^{2}$, is small, one can calculate $a_{p_{p h y s . \alpha}}^{\dagger}$ in the vicinity of $a_{p_{\alpha}}^{\dagger}$ and neglect all terms of order higher then $g^{2}$. The result is

$$
\begin{aligned}
& a_{\lambda p_{\text {phys. }}}^{\dagger}=a_{\lambda p_{\alpha}}^{\dagger} \\
& +g^{2} \frac{\delta \tilde{m}_{0}^{2}}{p_{\alpha}^{+}}\left(\frac{\sin \alpha}{2} \frac{\partial}{\partial p^{2}}-\sin ^{2} \frac{\alpha}{2} \frac{\partial}{\partial p^{+}}\right) a_{\lambda p_{\alpha}}^{\dagger} .
\end{aligned}
$$

The operator correction of order $g^{2}$ in Eq. (4.19) compensates the operator that acts on the vacuum state, $|0\rangle$, in Eq. (4.18). Since the state components $\left|\Psi_{1}\right\rangle$ and $\left|\Psi_{2}\right\rangle$ have wave-functions of order $g$ and $g^{2}$, one can simply replace $p_{\text {phys. }}$. with $p$ and $p_{\text {phys. } \alpha}$ with $p_{\alpha}$ in these terms without any change in the wave-functions. However, in the term with wave-function order $g^{0}=1$, the difference between $p_{\alpha}$ and $p_{\text {phys. } \alpha}$ in rotated effective creation operators does matter for terms of order $g^{2}$ in the rotated eigenstate. The second order difference turns out to be entirely contained in the mass correction. This difference is correctly restored by the exponentiated generator $J_{\lambda}^{1}$ through contractions with two particle components of the physical states. The origin of the second term on the right-hand side of Eq. (4.18) is thus explained.

In summary, the derived angular momentum operator $J_{\lambda}^{1}$ generates correct rotations of single physical particle states in all terms of second order perturbation theory. In the same way one calculates expressions for other quantum Poincaré transformations and verifies with the same accuracy that they transform one particle states in agreement with requirements of special relativity.

\section{CONCLUSION}

The Poincaré group generators obtained from scalar quantum field theory in second-order renormalization group procedure for effective particles, fulfill all Poincaré algebra commutation relations in terms of order $1, g$ and $g^{2}$. For example, the algebra includes the hard-to-satisfy relation in LF dynamics,

$$
\left[J_{\lambda}^{i}, J_{\lambda}^{j}\right]=i \epsilon_{i j k} J_{\lambda}^{k}+o\left(g^{3}\right) .
$$

This relation was not easy to derive in LF dynamics because two angular momentum operators depend on interactions. On the other hand, the dynamical parts of $J_{\lambda}^{i}$, $i=1,2$, are necessary to produce the correct mass values and corresponding terms in rotated states. The full Poincaré algebra is satisfied with the same accuracy for arbitrary values of the renormalization group parameter $\lambda$.

Since $\lambda$ equals to the width of vertex form factors in the renormalized Hamiltonians $H_{\lambda}\left(q_{\lambda}\right)$, the construction described in this work links a relativistic local field theory with a smooth quantum mechanics of effective particles in the Fock space. For $\lambda=\mu$ that matches a physically relevant scale, observable quantities are expected to be most conveniently calculable from the Schrödinger equations with corresponding $H_{\mu}\left(q_{\mu}\right)$, using computers that 
need to handle only a limited set of basis states required to cover the range of momentum scales around $\mu$. The new result of this work is that the Poincaré symmetry of these observables can be sought in a sequence of well defined approximations based on the renormalization group procedure for effective particles, too. The key remaining question of the scalar example, studied here only up to terms of order $g^{2}$, is whether it can indeed be systematically improved as expected by including terms of order $g_{\lambda}^{n}$ with $n>2$. The difference between series expansion in powers of $g$ and expansion in powers of $g_{\lambda}$, will appear first in terms of order $g^{3}$.

Exponentiation of the effective algebra gives operators that represent finite Poincaré transformations. They all turn out to change physical single-particle states in agreement with requirements of special relativity. This result includes spatial rotations in the LF dynamics and was also verified up to terms of order $g^{2}$. No anomalies were found. All non-canonical terms obtained from solutions of renormalization group equations had to be included in the Hamiltonian of finite $\lambda$ and in the corresponding angular momentum operators to obtain this result. It is natural to expect that similar but more complicated terms have to be included in effective theories of quarks and gluons when one tries to interpret data for scale-dependent hadronic observables, such as spin structure of the proton [8], in terms of constituents. It is therefore certainly interesting to carry out bound state studies using second order effective Hamiltonians in the scalar theory, since they can provide basic intuition about interaction-dependent effects in the angular momentum of partons. However, the basic study must be considerably extended to incorporate spin of individual particles in order to become directly applicable to LF QCD. The extension requires solution to problems with additional small- $p^{+}$singularities that characterize gauge bosons [9] The latter problem has to be solved in order to explicitly construct boosted and rotated hadronic bound states in the Fock space of effective quarks and gluons. Such construction could help, for example, in interpreting rel- ativistic partial wave analysis of decays of exotic hybrid states.

Although the original perturbative analysis of hadronic processes with high momentum transfers 10 raises hopes for a constituent picture of hadrons to be valid in the available range of energies, one has to deal with the fact that at the low energy end initial bound state studies 11] in LF QCD indicate that spin multiplets of excited states in spectra of approximate effective Hamiltonians may contain splittings that violate rotational symmetry and demand understanding. If effective generators of rotations in LF QCD with small- $\lambda$ were available, one could transform members of a broken multiplet and determine what kind of components are generated. The latter could be compared with the structure of eigenstates in the broken multiplet. Such comparison could help in finding out what effective Fock components are missing in the approximate calculations.

\section{APPENDIX A: BARE POINCARÉ GENERATORS}

LF co-ordinates are defined to be $x^{ \pm}=x^{0} \pm x^{3}$ and $x^{\perp}=\left(x^{1}, x^{2}\right)$, with a scalar product of two four-vectors equal $a b=a^{+} b^{-} / 2+a^{-} b^{+} / 2-a^{\perp} b^{\perp}$. Definitions (2.3) and (2.4) lead to the following kinematical generators,

$$
\begin{aligned}
P^{+} & =\int[p] p^{+} a_{p}^{\dagger} a_{p} \\
P^{i} & =\int[p] p^{i} a_{p}^{\dagger} a_{p} \\
M^{+-} & =-2 i \int[p] p^{+} \frac{\partial a_{p}^{\dagger}}{\partial p^{+}} a_{p}, \\
M^{+j} & =i \int[p] p^{+} \frac{\partial a_{p}^{\dagger}}{\partial p^{j}} a_{p}, \\
M^{12} & =i \int[p]\left(p^{1} \frac{\partial a_{p}^{\dagger}}{\partial p^{2}} a_{p}-p^{2} \frac{\partial a_{p}^{\dagger}}{\partial p^{1}} a_{p}\right),
\end{aligned}
$$

and dynamical generators,

$$
\begin{aligned}
P^{-} & =P_{0}^{-}+g P_{1}^{-}=\int[p] p^{-} a_{p}^{\dagger} a_{p}+\frac{1}{2} g \int[123] \tilde{\delta}\left(a_{1}^{\dagger} a_{2}^{\dagger} a_{3}+h . c .\right) \\
M^{-j} & =M_{0}^{-j}+g M_{1}^{-j} \\
& =i \int[p]\left(p^{-} \frac{\partial a_{p}^{\dagger}}{\partial p^{j}} a_{p}+2 p^{j} \frac{\partial a_{p}^{\dagger}}{\partial p^{+}} a_{p}\right)+\left[\frac{i}{2} g \int[123]\left(\frac{\partial}{\partial p_{3}^{j}} \tilde{\delta}\right) a_{1}^{\dagger} a_{2}^{\dagger} a_{3}+\text { h.c. }\right] .
\end{aligned}
$$

The symbol $\tilde{\delta}$, which appears also in Eq. (2.6), always denotes the three-momentum conservation Dirac $\delta$-function times $16 \pi^{3}$. For example, in Eq. (A7), $\tilde{\delta}=16 \pi^{3} \delta\left(p_{3}^{+}-\right.$ $\left.p_{12}^{+}\right) \delta\left(p_{3}^{1}-p_{12}^{1}\right) \delta\left(p_{3}^{2}-p_{12}^{2}\right)$, where $p_{12}^{+, \perp}=p_{1}^{+, \perp}+p_{2}^{+, \perp}$. The arguments of $\tilde{\delta}$ are always given by $\sum_{i \in \text { out }} p_{i}-\sum_{j \in \text { in }} p_{j}$, where the momenta in the set in are always those of particles annihilated in the interaction and momenta in the set out are always those of particles created in the interaction. Arguments of $\tilde{\delta}$ are written explicitly only if 
needed. The symbol $[p]$ denotes integration measure,

$$
[p]=\frac{d^{2} p^{\perp} d p^{+}}{16 \pi^{3} p^{+}} \theta\left(p^{+}\right) .
$$

The first order term in the regularization-dependent commutator in Eq. (2.9), is

$$
B_{\Delta 1}^{j}=\frac{i}{2} \int[123]\left[\sum_{l=1}^{2}\left(p_{l}^{-} \frac{\partial}{\partial p_{l}^{j}}+2 p_{l}^{j} \frac{\partial}{\partial p_{l}^{+}}\right) r_{\Delta}(12)\right] \tilde{\delta} a_{1}^{\dagger} a_{2}^{\dagger} a_{3}-\text { h.c. }
$$

and the second order term is

$$
\begin{aligned}
B_{\Delta 2}^{j}= & i \int[1234] \frac{\theta\left(p_{1}^{+}-p_{3}^{+}\right)}{p_{1}^{+}-p_{3}^{+}}\left\{\left.\frac{\partial}{\partial p^{j}}\left[r_{\Delta}(2 p) r_{\Delta}(3 p)\right]\right|_{p=p_{1}-p_{3}}\right\} \tilde{\delta} a_{1}^{\dagger} a_{2}^{\dagger} a_{3} a_{4} \\
& +\left\{\frac{i}{2} \int[1234] \frac{1}{p_{12}^{+}} r_{\Delta}(12)\left[\left.\frac{\partial}{\partial p^{j}} r_{\Delta}(3 p)\right|_{p=p_{1}+p_{2}}\right] \tilde{\delta} a_{1}^{\dagger} a_{2}^{\dagger} a_{3}^{\dagger} a_{4}-\text { h.c. }\right\}
\end{aligned}
$$

The commutator $B_{\Delta}^{12}$ of Eq. (2.10), has a similar structure to $B_{\Delta}^{j}$. All these terms would vanish if the regularization factors were absent.

\section{APPENDIX B: RENORMALIZATION GROUP FOR PARTICLES}

Starting from the regularized Hamiltonian $H_{\Delta}$ with counterterms, one constructs a family of annihilation and creation operators, parametrized by $\lambda$. Operators $a$ and $a^{\dagger}$ in $H_{\Delta}$ correspond to $\lambda=\infty$. When $H_{\Delta}$ is re-written in terms of $a_{\lambda}$ and $a_{\lambda}^{\dagger}$, the coefficients in front of products of these operators depend on $\lambda$, but the Hamiltonian remains unchanged, which is expressed as $H_{\lambda}\left(q_{\lambda}\right)=H_{\Delta}(q)$. Thus, all Hamiltonians with different $\lambda$ s are equal and for any $\lambda_{1}$ and $\lambda_{2}$,

$$
H_{\lambda_{1}}\left(q_{\lambda_{1}}\right)=H_{\lambda_{2}}\left(q_{\lambda_{2}}\right)
$$

Introducing $\mathcal{H}_{\lambda}=H_{\lambda}(q)$, which has the same coefficients in front of $q \mathrm{~s}$ as $H_{\lambda}\left(q_{\lambda}\right)$ has in front of $q_{\lambda} \mathrm{s}$, using the equality of Hamiltonians (B1) and unitary relation (3.1), one obtains

$$
\mathcal{H}_{\lambda}=\mathcal{U}_{\lambda}^{\dagger} \mathcal{H}_{\infty} \mathcal{U}_{\lambda}
$$

where all operators are written in terms of constant, i.e. independent of $\lambda$, bare operators $q . H_{\lambda}\left(q_{\lambda}\right)$ is obtained from $\mathcal{H}_{\lambda}$ by replacing $q \equiv q_{\infty}$ with $q_{\lambda}$.

Since $H_{\lambda}$ is to contain vertex form facthors that limit changes of kinetic energy of interacting effective particles, one assumes $H_{\lambda}$ to have the form

$$
H_{\lambda}=F_{\lambda}\left[G_{\lambda}\right]
$$

$F_{\lambda}$ is a linear operation that changes any operator $\hat{O}_{\lambda}$,

$$
\hat{O}_{\lambda}=\int[1 . . n n+1 . . m] \tilde{\delta} v(1, . ., m) a_{\lambda 1}^{\dagger} \cdots a_{\lambda n}^{\dagger} a_{\lambda n+1} \cdots a_{\lambda m}
$$

by introducing the form factor $f_{\lambda}$,

$$
F_{\lambda} \hat{O}_{\lambda}=\int[1 . . n n+1 . . m] \tilde{\delta} f_{\lambda} v(1, . ., m) a_{\lambda 1}^{\dagger} \cdots a_{\lambda n}^{\dagger} a_{\lambda n+1} \cdots a_{\lambda m}
$$

where

$$
f_{\lambda}=\exp \left[-\frac{\left(\mathcal{M}_{\text {in }}^{2}-\mathcal{M}_{\text {out }}^{2}\right)^{2}}{\lambda^{4}}\right]
$$

$\mathcal{M}_{\text {in }}^{2}=\left(p_{1}+\ldots+p_{n}\right)^{2}$, and $\mathcal{M}_{\text {out }}^{2}=\left(p_{n+1}+\ldots+p_{m}\right)^{2}$. In an abbreviated notation, one writes $H=f G$. One introduces then $\mathcal{G}_{\lambda}$, which is connected with $G_{\lambda}$ in the same way as $\mathcal{H}_{\lambda}$ is with $H_{\lambda}$. $\mathcal{G}_{\lambda}$ is divided into two parts, 
$\mathcal{G}_{\lambda}=\mathcal{G}_{0}+\mathcal{G}_{I \lambda}$, where $\mathcal{G}_{0}=\mathcal{G}_{\lambda}(g=0)$. $\mathcal{G}_{I \lambda}$ is assumed to satisfy the following differential equation (see e.g. [3]),

$$
\frac{d}{d \lambda} \mathcal{G}_{I \lambda}=\left[f \mathcal{G}_{I \lambda},\left\{\frac{d}{d \lambda}(1-f) \mathcal{G}_{I \lambda}\right\}_{\mathcal{G}_{0}}\right],
$$

where for any operator $\hat{O}, \hat{Q}=\{\hat{O}\}_{\mathcal{G}_{0}}$ indicates a solution of equation $\left[\hat{Q}, \mathcal{G}_{0}\right]=\hat{O}$. Equation (B7) guarantees that perturbation theory is free from small energy denominators, effective interactions are connected, they possess required cluster properties and preserve all kinematical symmetries of LF dynamics. To solve Eq. (B7) with accuracy to terms of order $g^{2}$, one writes

$$
\mathcal{G}_{I \lambda}=g \tau_{1}+g^{2} \tau_{2}+\cdots .
$$

Equation (B7) implies

$$
\begin{aligned}
\tau_{1}^{\prime} & =0 \\
\tau_{2}^{\prime} & =\left[\left\{f^{\prime} \tau_{1}\right\}, f \tau_{1}\right]=\left(\left\{f^{\prime}\right\} f-f\left\{f^{\prime}\right\}\right)\left[\tau_{1} \tau_{1}\right] .
\end{aligned}
$$

Integrating $(\mathrm{B} 9)$, one obtains $\tau_{\lambda 1}=\tau_{\infty 1}$, which means that

$$
P_{1 \lambda}^{-}=f_{\lambda} \tau_{\lambda 1}\left(q \rightarrow q_{\lambda}\right)=\frac{1}{2} \int[123] \tilde{\delta} f_{\lambda} a_{\lambda 1}^{\dagger} a_{\lambda 2}^{\dagger} a_{\lambda 3}+\text { h.c. } .
$$

In contrast to ( $\mathrm{A} 6), a^{\dagger}$ and $a$ in the effective Hamiltonian correspond to the scale $\lambda$. However, it is sometimes convenient to omit the subscript $\lambda$ in various symbols for simplicity of notation.

One introduces

$$
\begin{aligned}
& \tau_{1}=\alpha_{21}+\alpha_{12}, \\
& \tau_{2}=\beta_{11}+\beta_{31}+\beta_{13}+\beta_{22}
\end{aligned}
$$

$\alpha_{21}$ denotes the part of $\tau_{1}$ that contains two creation operators and one annihilation operator. Analogous convention is used in all subscripts. Equation (B10) gives,

$$
\begin{aligned}
\beta_{\lambda 11} & =2 \mathcal{F}_{2 \lambda}\left[\alpha_{12} \alpha_{21}\right]_{11}+\beta_{\infty 11}, \\
\beta_{\lambda 31} & =2 \mathcal{F}_{2 \lambda}\left[\alpha_{21} \alpha_{21}\right]_{31}, \\
\beta_{\lambda 13} & =2 \mathcal{F}_{2 \lambda}\left[\alpha_{12} \alpha_{12}\right]_{13}, \\
\beta_{\lambda 22} & =\mathcal{F}_{2 \lambda}\left[\alpha_{21} \alpha_{12}+4 \alpha_{12} \alpha_{21}\right]_{22},
\end{aligned}
$$

where

$$
\mathcal{F}_{2 \lambda}=\int_{\lambda}^{\infty} d s\left(\left\{f_{s}^{\prime}\right\} f_{s}-f_{s}\left\{f_{s}^{\prime}\right\}\right)
$$

For $f_{\lambda}=\exp \left(-a b^{2} / \lambda^{4}\right)$, see [3],

$$
\mathcal{F}_{2 \lambda}(a, b, c)=\frac{p_{b a}^{+} b a+p_{b c}^{+} b c}{b a^{2}+b c^{2}}\left(f_{a b} f_{b c}-1\right),
$$

where $a b=\mathcal{M}_{a}^{2}-\mathcal{M}_{b}^{2}$ and $a$ and $b$ are the corresponding configurations of particle momenta. In Section III and Appendix C, factors $\mathcal{F}_{2 \lambda}^{22 s}(1234), \mathcal{F}_{2 \lambda}^{22 e x}(1234)$ and

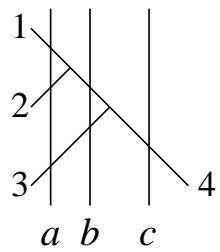

i)

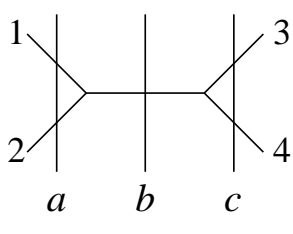

ii)

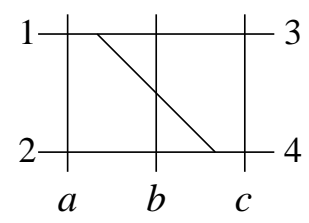

iii)
FIG. 4: Graphical illustration of interaction terms with factors i) $\mathcal{F}_{2 \lambda}^{31}(1234)$; ii) $\mathcal{F}_{2 \lambda}^{22 s}(1234)$; iii) $\mathcal{F}_{2 \lambda}^{22 e x}(1234)$.

$\mathcal{F}_{2 \lambda}^{31}(1234)$ correspond to the configurations shown in Fig. 4. Functions $V_{\lambda}^{22}$ and $V_{\lambda}^{31}$ in Eq. (3.5) for $P_{\lambda 2}^{-}$, are given in terms of $\mathcal{F}_{2 \lambda}$ by relations:

$$
\begin{aligned}
V_{\lambda}^{22} & =\frac{1}{4 p_{12}^{+}} \mathcal{F}_{2 \lambda}^{22 s}+\frac{\theta\left(p_{1}^{+}-p_{3}^{+}\right)}{p_{1}^{+}-p_{3}^{+}} \mathcal{F}_{2 \lambda}^{22 e x}, \\
V_{\lambda}^{31} & =\frac{1}{2 p_{12}^{+}} \mathcal{F}_{2 \lambda}^{31} .
\end{aligned}
$$

Functions $\tilde{V}_{\lambda}^{j 22}$ and $\tilde{V}_{\lambda}^{j 31}$ in Eq. (3.7) for $M_{2 \lambda}^{-j}$, are given by:

$$
\begin{aligned}
& \tilde{V}_{\lambda}^{j 22}=\left.\frac{\theta\left(p_{1}^{+}-p_{3}^{+}\right)}{p_{1}^{+}-p_{3}^{+}} \frac{\partial}{\partial p^{j}}\left(f_{2 p} f_{3 p}\right)\right|_{p=p_{1}-p_{3}}, \\
& \tilde{V}_{\lambda}^{j 31}=\left.\frac{1}{2 p_{12}^{+}} f_{12} \frac{\partial}{\partial p^{j}} f_{3 p}\right|_{p=p_{1}+p_{2}} .
\end{aligned}
$$

The subscripts $i j$ of the form factors $f_{i j}$ indicate that one of the invariant masses in the definition (B6) is given by $\left(p_{i}+p_{j}\right)^{2}$, while the other by $m^{2}$.

Second order terms involve mass renormalization in $\beta_{\lambda 11}$ that equals

$$
\beta_{\lambda 11}=\int[p] \frac{\delta m_{\lambda}^{2}}{p^{+}} a_{p}^{\dagger} a_{p},
$$

where, according to (B14),

$$
\delta m_{\lambda}^{2}=\delta m_{\infty}^{2}+\frac{1}{32 \pi^{2}} \int_{0}^{1} d x \int_{\mathcal{M}_{0}^{2}}^{\infty} d \mathcal{M}^{2} \frac{f_{\lambda}^{2}-1}{\mathcal{M}^{2}-m^{2}} r_{\Delta}^{2} .
$$

The counterterm contribution, $\delta m_{\infty}^{2}$, should remove $\Delta$ dependence from the Hamiltonian in the limit $\Delta \rightarrow \infty$. Hence,

$$
\delta m_{\infty}^{2}=\frac{1}{32 \pi^{2}} \ln \frac{\Delta}{m}+c .
$$

The arbitrary constant $c$ is a finite part of the counterterm and may be chosen in such a way that for some value of $\lambda$, say $\lambda_{0}$, mass squared in the effective Hamiltonian equals $m^{2}+g^{2} \delta m_{0}^{2}$. The value of $\delta m_{0}^{2}$ is determined by comparing theoretical predictions with experimental 
data. Then,

$$
\delta m_{\infty}^{2}=\delta m_{0}^{2}-\frac{1}{32 \pi^{2}} \int_{0}^{1} d x \int_{\mathcal{M}_{0}^{2}}^{\infty} d \mathcal{M}^{2} \frac{f_{\lambda_{0}}^{2}-1}{\mathcal{M}^{2}-m^{2}} r_{\Delta}^{2}
$$

Combining Eqs. (B25) and ( $\mathrm{B} 27)$, and taking the limit $\Delta \rightarrow \infty$, one obtains

$$
\delta m_{\lambda}^{2}=\delta m_{0}^{2}+\frac{1}{32 \pi^{2}} \int_{0}^{1} d x \int_{\mathcal{M}_{0}^{2}}^{\infty} d \mathcal{M}^{2} \frac{f_{\lambda}^{2}-f_{\lambda_{0}}^{2}}{\mathcal{M}^{2}-m^{2}}
$$

\section{APPENDIX C: CONNECTION BETWEEN BARE AND EFFECTIVE PARTICLES}

It follows from Eqs. (3.1) and (B7) that

$$
\frac{d}{d \lambda} \mathcal{U}_{\lambda}=\mathcal{U}_{\lambda} \frac{d}{d \lambda}\left\{(1-f) \mathcal{G}_{I \lambda}\right\}_{\mathcal{G}_{0}}
$$

The initial condition is provided at $\lambda=\infty: \mathcal{U}_{\infty}=1$. Assuming an infinitesimally small $g, \mathcal{U}_{\lambda}$ may be found using series expansion in powers of $g$, since $\mathcal{U}_{\lambda}(g=0)=$ 1 , and $\mathcal{G}_{I \lambda}$ is made of terms of order $g^{n}$ with $n \geq 1$. So,

$$
\mathcal{U}_{\lambda}=1+g u_{\lambda 1}+g^{2} u_{\lambda 2}+\cdots .
$$

Equation (C1) provides

$$
u_{\lambda 1}=\left\{\left(1-f_{\lambda}\right) \mathcal{G}_{1}\right\},
$$

and

$$
u_{\lambda 2}=\frac{1}{2} u_{\lambda 1}^{2}+v_{\lambda 2}
$$

where

$$
v_{\lambda 2}=\left\{\left(1-f_{\lambda}\right) \mathcal{G}_{2}\right\}+\frac{1}{2} \int_{\infty}^{\lambda} d s\left[u_{s 1}, u_{s 1}^{\prime}\right]
$$

Using this solution for $\mathcal{U}_{\lambda}$ and Eq. (3.1), one can write $q_{\infty}$ in terms of $q_{\lambda}$ as follows.

$$
\begin{aligned}
q_{0} & =q_{\lambda} \\
q_{1} & =\left[q_{\lambda}, u_{\lambda 1}\right] \\
q_{2} & =\left[q_{\lambda}, v_{\lambda 2}\right]+\frac{1}{2}\left[\left[q_{\lambda}, u_{\lambda 1}\right], u_{\lambda 1}\right]
\end{aligned}
$$

In full detail, see Fig. 5,

$$
\begin{aligned}
a_{1 p}^{\dagger}=\int[12] & {\left[-\frac{1}{2} \tilde{\delta}\left(p-p_{12}\right) r_{12} \frac{1-f_{12}}{p^{-}-p_{12}^{-}} a_{\lambda 1}^{\dagger} a_{\lambda 2}^{\dagger}\right.} \\
& \left.+\tilde{\delta}\left(p_{2}-p_{1 p}\right) r_{1 p} \frac{1-f_{1 p}}{p_{2}^{-}-p_{1 p}^{-}} a_{\lambda 2}^{\dagger} a_{\lambda 1}\right]
\end{aligned}
$$

and

$$
\begin{aligned}
a_{2 p}^{\dagger}= & -\frac{1}{64 \pi^{2}} \int_{0}^{1} d x \int_{\mathcal{M}_{0}^{2}}^{\infty} d \mathcal{M}^{2} \frac{\left(1-f_{\lambda}\right)^{2}}{\left(\mathcal{M}^{2}-m^{2}\right)^{2}} r_{\Delta}^{2} a_{\lambda p}^{\dagger} \\
& -\int[123]\left\{\frac{1}{2} r_{12} r_{(12) 3} \frac{1}{p_{12}^{+}} a_{+}^{31}(123 p) \tilde{\delta}\left(p-p_{123}\right) a_{\lambda 1}^{\dagger} a_{\lambda 2}^{\dagger} a_{\lambda 3}^{\dagger}\right. \\
& -\left[r_{1 p} r_{(1 p) 2} \frac{1}{p_{1 p}^{+}} a_{-}^{31}(p 123)+\frac{1}{2} r_{12} r_{(12) p} \frac{1}{p_{12}^{+}} a_{+}^{31}(12 p 3)\right] \tilde{\delta}\left(p_{3}-p_{12 p}\right) a_{\lambda 3}^{\dagger} a_{\lambda 1} a_{\lambda 2} \\
& +\left[r_{3(1-3)} r_{2(p-2)} \frac{\theta\left(p_{1}^{+}-p_{3}^{+}\right)}{p_{1}^{+}-p_{3}^{+}} a_{+}^{22 e x}(123 p)+r_{p(1-p)} r_{2(3-2)} \frac{\theta\left(p_{1}^{+}-p^{+}\right)}{p_{1}^{+}-p^{+}} a_{-}^{22 e x}(12 p 3)\right. \\
& \left.\left.+r_{12} r_{3 p} \frac{1}{2 p_{12}^{+}} a_{+}^{22 s}(123 p)\right] \tilde{\delta}\left(p_{3 p}-p_{12}\right) a_{\lambda 1}^{\dagger} a_{\lambda 2}^{\dagger} a_{\lambda 3}\right\}
\end{aligned}
$$

where

$$
a_{ \pm}=\mathcal{B} \pm \mathcal{C}
$$

$$
\begin{aligned}
& \mathcal{B}=p_{a c}^{+} \frac{1-f_{a c}}{c a} \mathcal{F}_{2}(a, b, c) \\
& -\frac{1}{2} \frac{p_{a b}^{+} p_{b c}^{+}}{b a b c}\left[f_{a b}-f_{b c}-\frac{b a^{2}-b c^{2}}{b a^{2}+b c^{2}}\left(f_{a b} f_{b c}-1\right)\right]
\end{aligned}
$$



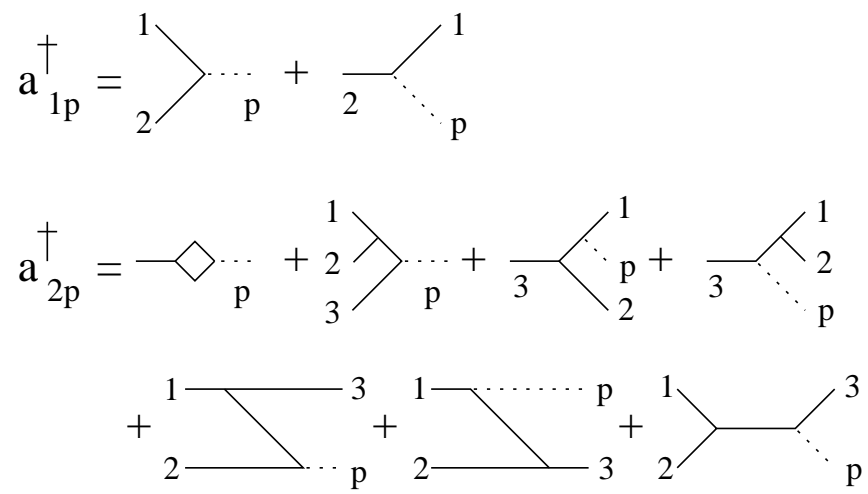

FIG. 5: Numbered lines stand for creation and annihilation operators of effective particles, and dots indicate how momentum $p$ enters Eqs. (C9) and ( $\mathrm{C} 10)$.

$$
\mathcal{C}=\frac{1}{2} \frac{p_{a b}^{+} p_{b c}^{+}}{b a b c}\left(1-f_{a b}\right)\left(1-f_{b c}\right)
$$

Indices in parenthesis in $r_{i(j k)}$, or $r_{i(j-k)}$, mean that $\kappa^{\perp}$ is calculated as a relative momentum of particle $i$ and the second particle with momentum $p_{j}^{+, \perp}+p_{k}^{+, \perp}$, or $p_{j}^{+, \perp}-$ $p_{k}^{+, \perp}$, respectively. The arguments of $a_{ \pm}(1234)$ refer to particle momenta in the configurations $a, b$ and $c$, which are labeled using the same convention as used for $\mathcal{F}_{2 \lambda}$ in Fig. 4.

Note that if the operator trajectories $a_{\lambda}$ and Poincaré generators at one finite value of $\lambda$ were known, there would be no need to trace the changes of the generators all the way back to a local theory. Unfortunately, they are not known and the construction developed here has to draw on the formal structure that a local theory implies through perturbation theory. An exact solution of Eq. (B7), if it existed, could extend the approach beyond perturbation theory. Virtually nothing is known about existence of such solutions, but if they existed, one should expect them to possess a rich mathematical structure.

\section{APPENDIX D: DYNAMICAL ROTATIONS}

This Appendix describes derivation and features of spatial rotation operators in LF dynamics on example of the rotation around one of the transverse axes. The operator $U(\alpha)$ for rotations around axis number 1 by angle $\alpha$, is calculated similarly to the $S$-matrix in old-fashioned perturbation theory, except that the angle $\alpha$ is analogous to a finite interval of time. One writes $U(\alpha)$ in the form

$$
U(\alpha)=W(\alpha) e^{-i \alpha J_{0 \lambda}^{1}}
$$

so that

$$
W(\alpha)=e^{-i \alpha J_{\lambda}^{1}} e^{i \alpha J_{0 \lambda}^{1}}
$$

$W(\alpha)$ satisfies the differential equation

$$
\frac{d}{d \alpha} W(\alpha)=-i W(\alpha) J_{I \lambda}^{1}(\alpha)
$$

where

$$
J_{I \lambda}^{1}(\alpha)=e^{-i \alpha J_{0 \lambda}^{1}} J_{I \lambda}^{1} e^{i \alpha J_{0 \lambda}^{1}},
$$

and $J_{I \lambda}^{1}=J_{\lambda}^{1}-J_{0 \lambda}^{1}=\left(M_{\lambda}^{-2}-M_{0 \lambda}^{-2}\right) / 2$. When the interaction is absent, $U(\alpha)=e^{-i \alpha J_{0 \lambda}^{1}}$ and $W(\alpha)=1$. For infinitesimally small coupling constants $g$, one can integrate Eq. D3) term by term in a power series in $g$. One obtains

$$
W(\alpha)=1+g W_{1}(\alpha)+g^{2} W_{2}(\alpha)+\cdots,
$$

where

$$
W_{1}(\alpha)=-i \int_{0}^{\alpha} d \beta J_{\lambda 1}^{1}(\beta),
$$

$$
\begin{aligned}
& W_{2}(\alpha)=W_{2}^{(11)}(\alpha)+W_{2}^{(2)}(\alpha) \\
& =(-i)^{2} \int_{0}^{\alpha} d \beta \int_{0}^{\beta} d \beta^{\prime} J_{\lambda 1}^{1}\left(\beta^{\prime}\right) J_{\lambda 1}^{1}(\beta)-i \int_{0}^{\alpha} d \beta J_{\lambda 2}^{1}(\beta) .
\end{aligned}
$$

\section{Terms independent of interactions}

Using relations

$$
\begin{aligned}
& e^{-i \alpha J_{0 \lambda}^{1}} a_{\lambda p}^{\dagger} e^{i \alpha J_{0 \lambda}^{1}}=a_{\lambda p_{\alpha}}^{\dagger}, \\
& e^{-i \alpha J_{0 \lambda}^{1}} a_{\lambda p} e^{i \alpha J_{0 \lambda}^{1}}=a_{\lambda p_{\alpha}}
\end{aligned}
$$

one obtains

$$
\begin{aligned}
& \left|\Psi_{\alpha 0}(p)\right\rangle=U_{0}(\alpha)\left|\Psi_{0}(p)\right\rangle \\
& =U_{0}(\alpha) a_{\lambda p}^{\dagger} U_{0}^{-1}(\alpha) U_{0}(\alpha)|0\rangle=a_{\lambda p_{\alpha}}^{\dagger}|0\rangle=\left|\Psi_{0}\left(p_{\alpha}\right)\right\rangle
\end{aligned}
$$

where

$$
\begin{aligned}
& p_{\alpha}^{+}=p^{+} \cos ^{2} \frac{\alpha}{2}+\frac{p^{\perp 2}+m^{2}}{p^{+}} \sin ^{2} \frac{\alpha}{2}+p^{2} \sin \alpha \\
& p_{\alpha}^{2}=p^{2} \cos \alpha-\frac{1}{2}\left(p^{+}-\frac{p^{\perp 2}+m^{2}}{p^{+}}\right) \sin \alpha \\
& p_{\alpha}^{1}=p^{1} .
\end{aligned}
$$

\section{First order terms}

According to (D6),

$$
W_{1}(\alpha)=-\frac{i}{2} \int_{0}^{\alpha} d \beta M_{\lambda 1}^{-2}(\beta),
$$


where

$$
M_{\lambda 1}^{-2}(\beta)=\frac{i}{2} \int\left[p_{1} p_{2} p_{3}\right] f_{\lambda}\left(\frac{\partial}{\partial p_{3}^{2}} \tilde{\delta}\right) a_{1 \beta}^{\dagger} a_{2 \beta}^{\dagger} a_{3 \beta}+\text { h.c. . }
$$

The subscript $\lambda$ in creation and annihilation operators is omitted in the above formula to make it more readable. The transformation of momenta is given by Eq. (D11) with $\alpha$ replaced by $\beta$. Changing variables from $p_{1}, p_{2}, p_{3}$ to $k_{1}=p_{1 \beta}, k_{2}=p_{2 \beta}, k_{3}=p_{3 \beta}$, one obtains

$$
M_{\lambda 1}^{-2}(\beta)=\frac{i}{2} \int\left[k_{1} k_{2} k_{3}\right] f_{\lambda}\left[\frac{\partial}{\partial p_{3}^{2}} \tilde{\delta}\left(k_{3 \hat{\beta}}-k_{1 \hat{\beta} 2 \hat{\beta}}\right)\right] a_{\lambda 1}^{\dagger} a_{\lambda 2}^{\dagger} a_{\lambda 3}+h . c .
$$

where the subscript $\hat{\beta}$ denotes the interaction-independent transformation of momenta $k^{+}$and $k^{\perp}$ that corresponds to inverse rotation to the rotation by the angle $\beta$.

$$
\frac{\partial}{\partial p_{3}^{2}}=\frac{1}{-k_{3}^{2} \mathrm{~s}+k_{3}^{+} \tilde{\mathrm{c}}^{2}+k_{3}^{-\tilde{\mathrm{s}}^{2}}}\left[\left(k_{3}^{+} \tilde{\mathrm{c}}^{2}-k_{3}^{-} \tilde{\mathrm{s}}^{2}\right) \frac{\partial}{\partial k_{3}^{2}}+2 \tilde{\mathrm{s}}\left(k_{3}^{+} \tilde{\mathrm{c}}-k_{3}^{2} \tilde{\mathrm{s}}\right) \frac{\partial}{\partial k_{3}^{+}}\right],
$$

where $\mathrm{s} \equiv \sin \beta, \tilde{\mathrm{s}} \equiv \sin \beta / 2, \mathrm{c} \equiv \cos \beta, \tilde{\mathrm{c}} \equiv \cos \beta / 2$.

Note that the derivative $\partial / \partial p_{3}^{2}$, in Eq. (D14), acts only on the $\delta$-function factor, $\delta\left(k_{3 \hat{\beta}}^{2}-k_{1 \hat{\beta} 2 \hat{\beta}}^{2}\right)$. Therefore, expression (D15) for $\partial / \partial p_{3}^{2}$ inside (D14) is equivalent to

$$
\frac{\partial}{\partial p_{3}^{2}}=\frac{1}{-\left(k_{3}^{2}-k_{12}^{2}\right) \mathrm{s}+\frac{1}{2}\left(k_{3}^{+}-k_{12}^{+}-k_{3}^{-}+k_{12}^{-}\right) \mathrm{c}} \frac{d}{d \beta} .
$$

The denominator may be simplified thanks to the factor $\delta\left(k_{3 \hat{\beta}}^{+}-k_{1 \hat{\beta} 2 \hat{\beta}}^{+}\right)$, which implies that $-\left(k_{3}^{2}-k_{12}^{2}\right) \mathrm{s}+\left(k_{3}^{+}-\right.$ $\left.k_{12}^{+}-k_{3}^{-}+k_{12}^{-}\right) \mathrm{c} / 2=-\left(k_{3}^{+}+k_{3}^{-}-k_{12}^{+}-k_{12}^{-}\right) / 2$. This way the dependence on $\beta$ is shown to be fully contained in the arguments of the $\delta$-functions, and

$$
\begin{aligned}
\frac{d}{d \beta} & {\left[\delta\left(k_{3 \hat{\beta}}^{2}-k_{1 \hat{\beta} 2 \hat{\beta}}^{2}\right)\right] \delta\left(k_{3 \hat{\beta}}^{+}-k_{1 \hat{\beta} 2 \hat{\beta}}^{+}\right) } \\
& =\frac{d}{d \beta}\left[\delta\left(k_{3 \hat{\beta}}^{2}-k_{1 \hat{\beta} 2 \hat{\beta}}^{2}\right) \delta\left(k_{3 \hat{\beta}}^{+}-k_{1 \hat{\beta} 2 \hat{\beta}}^{+}\right)\right]-\delta\left(k_{3 \hat{\beta}}^{2}-k_{1 \hat{\beta} 2 \hat{\beta}}^{2}\right) \frac{d}{d \beta}\left[\delta\left(k_{3 \hat{\beta}}^{+}-k_{1 \hat{\beta} 2 \hat{\beta}}^{+}\right)\right] .
\end{aligned}
$$

The second term on the right-hand side in the above equation gives no contribution because $d\left[\delta\left(k_{3 \hat{\beta}}^{+}-k_{1 \hat{\beta} 2 \hat{\beta}}^{+}\right)\right] / d \beta$ is proportional to the argument of $\delta\left(k_{3 \hat{\beta}}^{2}-k_{1 \hat{\beta} 2 \hat{\beta}}^{2}\right)$. So, the differentiation over $\beta$ may be extended to the entire factor $\tilde{\delta}\left(k_{3 \hat{\beta}}-k_{1 \hat{\beta} 2 \hat{\beta}}\right)$.

It follows that,

$$
M_{\lambda 1}^{-2}(\beta)=-i \frac{d}{d \beta} \int\left[k_{1} k_{2} k_{3}\right] \frac{f_{\lambda}}{k_{3 \hat{\beta}}^{-}-k_{1 \hat{\beta} 2 \hat{\beta}}^{-}} \tilde{\delta}\left(k_{3 \hat{\beta}}-k_{1 \hat{\beta} 2 \hat{\beta}}\right) a_{\lambda 1}^{\dagger} a_{\lambda 2}^{\dagger} a_{\lambda 3}+\text { h.c. }
$$

and one can integrate (D12) to obtain

$$
W_{1}(\alpha)=-\frac{1}{2} \int[123] f_{\lambda}\left[\frac{\tilde{\delta}\left(p_{3 \hat{\alpha}}-p_{1 \hat{\alpha} 2 \hat{\alpha}}\right)}{p_{3 \hat{\alpha}}^{-}-p_{1 \hat{\alpha} 2 \hat{\alpha}}^{-}}-\frac{\tilde{\delta}\left(p_{3}-p_{12}\right)}{p_{3}^{-}-p_{12}^{-}}\right] a_{\lambda 1}^{\dagger} a_{\lambda 2}^{\dagger} a_{\lambda 3}-\text { h.c. . }
$$

Then, the first order result for the rotated state reads

$$
\begin{array}{r}
\left|\Psi_{\alpha 1}(p)\right\rangle=U_{1}(\alpha)\left|\Psi_{0}(p)\right\rangle+U_{0}(\alpha)\left|\Psi_{1}(p)\right\rangle \\
=\frac{1}{2} \int[12] \frac{f_{\lambda}}{p_{\alpha}^{-}-p_{12}^{-}} \tilde{\delta}\left(p_{\alpha}-p_{12}\right)|12\rangle,
\end{array}
$$

as it should be.

\section{3. $\quad$ Second order terms}

The calculation of $\left|\Psi_{\alpha 2}(p)\right\rangle$ produces two parts. The first paragraph below lists the three-effective-particle contribution to the rotated state. The second paragraph shows one-effective-particle contribution, which includes renormalization. 


\section{a. Three-particle contributions}

The part of perturbative rotation operator $U(\alpha)$, which contributes to the three-particle component of the rotated state $\left|\Psi_{\alpha}(p)\right\rangle$ of order $g^{2}$, contains products of three creators and one annihilator for effective particles. For these terms, integration in $W_{2}(\alpha)$ is carried out in steps similar to the case of $W_{1}(\alpha)$ described earlier.

$$
W_{2}(\alpha)=W_{2}^{(2)}(\alpha)+W_{2}^{(11)}(\alpha)
$$

where

$$
\begin{aligned}
& W_{2}^{(2)}(\alpha)=-\frac{i}{2} \int_{0}^{\alpha} d \beta M_{\lambda 2}^{-2}(\beta) \\
& =-\int[1234] f_{\lambda}\left\{\frac{\tilde{\delta}\left(p_{4 \hat{\alpha}}-p_{1 \hat{\alpha} 2 \hat{\alpha} 3 \hat{\alpha})}\right.}{p_{4 \hat{\alpha}}^{-}-p_{1 \hat{\alpha} 2 \hat{\alpha} 3 \hat{\alpha}}^{-}} V_{\lambda}^{31}(\hat{\alpha})-\frac{\tilde{\delta}\left(p_{4}-p_{123}\right)}{p_{4}^{-}-p_{123}^{-}} V_{\lambda}^{31}\right\} a_{\lambda 1}^{\dagger} a_{\lambda 2}^{\dagger} a_{\lambda 3}^{\dagger} a_{\lambda 4} \\
& +\frac{1}{2} \int_{0}^{\alpha} d \beta \int[1234] \frac{\tilde{\delta}\left(p_{4 \hat{\beta}}-p_{1 \hat{\beta} 2 \hat{\beta} 3 \hat{\beta}}\right)}{p_{4 \hat{\beta}}^{-}-p_{1 \hat{\beta} 2 \hat{\beta} 3 \hat{\beta}}^{-}} \tilde{V}_{\lambda}^{j 31}(\hat{\beta}) a_{\lambda 1}^{\dagger} a_{\lambda 2}^{\dagger} a_{\lambda 3}^{\dagger} a_{\lambda 4}, \\
& W_{2}^{(11)}(\alpha)=-\frac{i}{2} \int_{0}^{\alpha} d \beta W_{1}(\beta) M_{\lambda 1}^{-2}(\beta) \\
& =\frac{1}{2} \int[1234] \frac{f_{12}}{m^{2}-\mathcal{M}_{12}^{2}}\left\{\frac{\tilde{\delta}\left(p_{4 \hat{\alpha}}-p_{1 \hat{\alpha} 2 \hat{\alpha} 3 \hat{\alpha}}\right)}{p_{4 \hat{\alpha}}^{-}-p_{1 \hat{\alpha} 2 \hat{\alpha} 3 \hat{\alpha}}^{-}} f_{(1 \hat{\alpha} 2 \hat{\alpha}) 3 \hat{\alpha}}-\frac{\tilde{\delta}\left(p_{4}-p_{123}\right)}{p_{4}^{-}-p_{123}^{-}} f_{(12) 3}\right. \\
& \left.-\frac{\tilde{\delta}\left(p_{4 \hat{\alpha}}-p_{(12) \hat{\alpha} 3 \hat{\alpha}}\right)}{p_{4}^{-}-p_{(12) \hat{\alpha} 3 \hat{\alpha}}^{-}} f_{(12) 3}+\frac{\tilde{\delta}\left(p_{4}-p_{123}\right)}{p_{4}^{-}-p_{(12) 3}^{-}} f_{(12) 3}\right\} a_{\lambda 1}^{\dagger} a_{\lambda 2}^{\dagger} a_{\lambda 3}^{\dagger} a_{\lambda 4} \\
& -\frac{1}{2} \int_{0}^{\alpha} d \beta \int[1234] \frac{\tilde{\delta}\left(p_{4 \hat{\beta}}-p_{1 \hat{\beta} 2 \hat{\beta} 3 \hat{\beta}}\right)}{p_{4 \hat{\beta}}^{-}-p_{1 \hat{\beta} 2 \hat{\beta} 3 \hat{\beta}}^{-}} \frac{f_{12}}{m^{2}-\mathcal{M}_{12}^{2}}\left[\frac{d}{d \beta} f_{(1 \hat{\beta} 2 \hat{\beta}) 3 \hat{\beta}}\right] a_{\lambda 1}^{\dagger} a_{\lambda 2}^{\dagger} a_{\lambda 3}^{\dagger} a_{\lambda 4} \text {. }
\end{aligned}
$$

The last term in $W_{2}^{(2)}(\alpha)$ in Eq. (D22) cancels the last term in $W_{2}^{(11)}(\alpha)$ in Eq. (D23). Collecting all contributions to $\left|\Psi_{\alpha 2}(p)\right\rangle$, one obtains

$$
\left|\Psi_{\alpha 2}(p)\right\rangle_{\text {tree }}=\left\{\left[U_{2}^{(2)}(\alpha)+U_{2}^{(11)}(\alpha)\right]\left|\Psi_{0}(p)\right\rangle+U_{1}(\alpha)\left|\Psi_{1}(p)\right\rangle+U_{0}(\alpha)\left|\Psi_{2}(p)\right\rangle\right\}_{\text {tree }}=\left|\Psi_{2}\left(p_{\alpha}\right)\right\rangle .
$$

\section{b. Renormalization of one-particle component}

Contributions to the one effective particle component of $\left|\Psi_{\alpha 2}(p)\right\rangle$, are

$$
\begin{aligned}
& \left|\Psi_{\alpha 2}(p)\right\rangle_{\text {loop }}=\left\{\left[U_{2}^{(2)}(\alpha)+U_{2}^{(11)}(\alpha)\right]\left|\Psi_{0}(p)\right\rangle+U_{1}(\alpha)\left|\Psi_{1}(p)\right\rangle\right\}_{\text {loop }} \\
& \left\{U_{2}^{(2)}(\alpha)\left|\Psi_{0}(p)\right\rangle\right\}_{\text {loop }}=\frac{\delta m_{\lambda}^{2}}{p_{\alpha}^{+}}\left(\frac{\sin \alpha}{2} \frac{\partial}{\partial p^{2}}-\sin ^{2} \frac{\alpha}{2} \frac{\partial}{\partial p^{+}}\right) a_{\lambda p_{\alpha}}^{\dagger}|0\rangle, \\
& \left\{U_{2}^{(11)}(\alpha)\left|\Psi_{0}(p)\right\rangle\right\}_{\text {loop }}=-\frac{\delta \tilde{m}_{\lambda}^{2}}{p_{\alpha}^{+}}\left(\frac{\sin \alpha}{2} \frac{\partial}{\partial p^{2}}-\sin ^{2} \frac{\alpha}{2} \frac{\partial}{\partial p^{+}}\right) a_{\lambda p_{\alpha}}^{\dagger}|0\rangle \\
& +\int[123] f_{\lambda}^{2} \frac{\tilde{\delta}\left(p_{3}-p_{12}\right)}{2\left(p_{3}^{-}-p_{12}^{-}\right)}\left[\frac{\tilde{\delta}\left(p-p_{1 \hat{\alpha} 2 \hat{\alpha}}\right)}{p^{-}-p_{1 \hat{\alpha} 2 \hat{\alpha}}^{-}}-\frac{\tilde{\delta}\left(p_{\alpha}-p_{12}\right)}{p_{\alpha}^{-}-p_{12}^{-}}\right] a_{\lambda 3}^{\dagger}|0\rangle \\
& \left\{U_{1}(\alpha)\left|\Psi_{1}(p)\right\rangle\right\}_{\text {loop }}=\int[123] f_{\lambda}^{2} \frac{\tilde{\delta}\left(p-p_{1 \hat{\alpha} 2 \hat{\alpha}}\right)}{2\left(p^{-}-p_{1 \hat{\alpha} 2 \hat{\alpha}}^{-}\right)}\left[\frac{\tilde{\delta}\left(p_{3 \hat{\alpha}}-p_{1 \hat{\alpha} 2 \hat{\alpha}}\right)}{p_{3 \hat{\alpha}}^{-}-p_{1 \hat{\alpha} 2 \hat{\alpha}}^{-}}-\frac{\tilde{\delta}\left(p_{3}-p_{12}\right)}{p_{3}^{-}-p_{12}^{-}}\right] a_{\lambda 3}^{\dagger}|0\rangle
\end{aligned}
$$


where

$$
\delta \tilde{m}_{\lambda}^{2}=\frac{1}{32 \pi^{2}} \int_{0}^{1} d x \int_{\mathcal{M}_{0}^{2}}^{\infty} d \mathcal{M}^{2} \frac{f_{\lambda}^{2}}{\mathcal{M}^{2}-m^{2}} .
$$

Combining these expressions, $\left(\left\{U_{1}(\alpha)\left|\Psi_{1}(p)\right\rangle\right\}_{\text {loop }}\right.$, Eq. (D28), cancels the last term in Eq. (D27)), one obtains

$$
\begin{aligned}
& \left|\Psi_{\alpha 2}(p)\right\rangle_{\text {loop }} \\
& =\frac{\delta m_{\lambda}^{2}-\delta \tilde{m}_{\lambda}^{2}}{p_{\alpha}^{+}}\left(\frac{\sin \alpha}{2} \frac{\partial}{\partial p^{2}}-\sin ^{2} \frac{\alpha}{2} \frac{\partial}{\partial p^{+}}\right) a_{\lambda p_{\alpha}}^{\dagger}|0\rangle .
\end{aligned}
$$

The complete second order term in Eq. (4.18) is given by the sum of the tree term with three effective particles, Eq. (D24), and the loop term with mass renormalization in the term with one effective particle, Eq. (D30).
[1] P. A. M. Dirac, Rev. Mod. Phys. 21, 392 (1949).

[2] K. Wilson, Phys. Rev. B140, 445 (1965); K. G. Wilson, Phys. Rev. D2, 1438 (1970).

[3] St. D. Głazek, Phys. Rev. D63, 116006 (2001); and references therein.

[4] St. D. Głazek and K. G. Wilson, Phys. Rev. D48, 5863 (1993); ibid. D49, 4214 (1994).

[5] See e.g. J. Kogut, L. Susskind, Phys. Rev. C8, 75 (1973); H. Leutwyler and J. Stern, Ann. of Phys. 112, 94 (1978); B.L.G. Bakker, L.A. Kondratyuk and M.V. Terent'ev, Nucl. Phys. B158 (1979) 497; F. Coester and W. N. Polyzou, Phys. Rev. D26, 1348 (1982).
[6] See e.g. S.-J. Chang, R. G. Root and T.-M. Yan Phys. Rev. D7, 1133 (1973).

[7] E. Wigner, Ann. of Math. 40, 149 (1939).

[8] A. Harindranath, A. Mukherjee, R. Ratabole, Phys. Rev. D63 045006 (2001).

[9] K. G. Wilson et al., Phys. Rev. D49, 6720 (1994).

[10] G. P. Lepage, S. J. Brodsky, Phys. Rev. D22, 2157 (1980).

[11] M. Brisudová and R. J. Perry, Phys. Rev. D54, 1831 (1996); B. H. Allen, R. J. Perry, Phys. Rev. D62, 025005 (2000). 